\title{
Minimum Required Detection Range for Detect and Avoid of Unmanned Aircraft Systems
}

Jared Kevin Wikle

Brigham Young University

Tim McLain

Brigham Young University - Provo, mclain@byu.edu

Randal W. Beard

Department of Electrical and Computer Engineering, Brigham Young University

Laith Rasmi Sahawneh

Brigham Young University - Provo

Follow this and additional works at: https://scholarsarchive.byu.edu/facpub

Part of the Mechanical Engineering Commons

\section{Original Publication Citation}

Wikle, Jared K., et al. "Minimum Required Detection Range for Detect and Avoid of Unmanned Aircraft Systems." Journal of Aerospace Information Systems (2017).

\section{BYU ScholarsArchive Citation}

Wikle, Jared Kevin; McLain, Tim; Beard, Randal W.; and Sahawneh, Laith Rasmi, "Minimum Required Detection Range for Detect and Avoid of Unmanned Aircraft Systems" (2017). Faculty Publications. 1972. https://scholarsarchive.byu.edu/facpub/1972 


\title{
Minimum Required Detection Range for
}

\section{Detect and Avoid of Unmanned Aircraft Systems}

\author{
Jared K. Wikle ${ }^{1}$ and Timothy W. McLain ${ }^{2}$ and Randal W. Beard ${ }^{3}$ \\ Brigham Young University, Provo, Utah, 84602 \\ Laith R. Sahawneh ${ }^{4}$ \\ University of Florida, Shalimar, Florida, 32579
}

For unmanned aircraft systems to gain full access to the National Airspace System, they must have the capability to detect and avoid other aircraft. To safely avoid another aircraft, an unmanned aircraft must detect the intruder aircraft with ample time and distance to allow the ownship to track the intruder, perform risk assessment, plan an avoidance path, and execute the maneuver. This paper describes two analytical methods for finding the minimum detection range to ensure that these detection and avoidance steps can be carried out. The first method, time-based geometric velocity vectors, includes the bank-angle dynamics of the ownship; whereas the second, geometric velocity vectors, assumes an instantaneous bank-angle maneuver. The solution using the first method must be found numerically, whereas the second has a closedform analytical solution. These methods are compared to two existing methods. The results show the time-based geometric velocity vectors approach is precise, the geometric velocity vectors approach is a good approximation under many conditions, and the two existing approaches are good approximations at large ownship speeds relative to the intruder speed, fast ownship bank-angle transients, and small ownship bank angles.

\footnotetext{
1 Research Assistant, Department of Mechanical Engineering.

2 Professor, Department of Mechanical Engineering. Associate Fellow AIAA.

3 Professor, Department of Electrical and Computer Engineering. Associate Fellow AIAA.

4 Postdoctoral Associate, AFRL/University of Florida Research and Engineering Education Facility. Student Member AIAA.
} 


\section{Nomenclature}

$$
\begin{aligned}
& a, b, c, d=\text { coefficients to a third-order polynomial in } \sin \theta_{\text {cpa }} \\
& d_{\mathrm{CPA}} \quad=\text { remaining head-on distance between the ownship and intruder at the closest } \\
& \text { point of approach }(\mathrm{m}) \\
& d_{\mathrm{MDR}}=\text { minimum detection range }(\mathrm{m}) \\
& d_{m} \quad=\text { distance between the ownship and intruder when the ownship starts } \\
& \text { maneuvering }(\mathrm{m}) \\
& d_{o}, d_{i}=\text { head-on distance traveled by the ownship and intruder }(\mathrm{m}) \\
& d_{o, c}, d_{i, c} \quad=\text { head-on distance traveled by the ownship and intruder during the } \\
& \text { computation time }(\mathrm{m}) \\
& d_{o, m}, d_{i, m} \quad=\text { head-on distance traveled by the ownship and intruder during the maneuver } \\
& \text { time }(\mathrm{m}) \\
& \bar{d}_{\mathrm{MDR}}=\text { minimum detection range with the slack parameter safety factor }(\mathrm{m}) \\
& g \quad=\text { gravitational constant }\left(\mathrm{m} / \mathrm{s}^{2}\right) \\
& K, \tau \quad=\text { general first-order system parameters } \\
& L \quad=\text { length of the ownship path during the maneuver }(\mathrm{m}) \\
& L_{3} \quad=\text { length of the ownship path during segment } 3(\mathrm{~m}) \\
& p \quad=\text { roll rate }(\mathrm{rad} / \mathrm{s}) \\
& \mathbf{p}_{o}, \mathbf{p}_{i} \quad=\text { position vector of the ownship and intruder in the right-handed } X-Y-Z \\
& \text { inertial reference frame }(\mathrm{m}) \\
& p_{x}, p_{y} \quad=X \text { and } Y \text { positions of aircraft }(\mathrm{m}) \\
& R_{\min }=\text { minimum turn radius }(\mathrm{m}) \\
& R_{s}, h_{s} \quad=\text { radius and height of safety volume }(\mathrm{m}) \\
& \dot{r} \quad=\text { range rate between the ownship and intruder aircraft }(\mathrm{m} / \mathrm{s}) \\
& s \quad=\text { independent variable used for Laplace representation } \\
& t \quad=\text { time }(\mathrm{s}) \\
& t_{c} \quad=\text { computation time }(\mathrm{s}) \\
& t_{\mathrm{DAA}}=\text { time required for detect-and-avoid operations }(\mathrm{s})
\end{aligned}
$$




$$
\begin{aligned}
& t_{m} \quad=\text { time to maneuver to the closest point of approach (s) } \\
& t_{t} \quad=\operatorname{turn} \text { time }(\mathrm{s}) \\
& t_{0}, t_{1}, t_{2}, t_{3}, t_{4}, t_{5} \quad=\text { times to change deflection angle of ailerons }(\mathrm{s}) \\
& v_{o}, v_{i}=\text { velocity of ownship and intruder aircraft }(\mathrm{m} / \mathrm{s}) \\
& \mathbf{v}_{o}, \mathbf{v}_{i} \quad=\text { velocity vector of the ownship and intruder in the right-handed } X-Y-Z \\
& \text { inertial reference frame }(\mathrm{m} / \mathrm{s}) \\
& v_{x}, v_{y}=X \text { and } Y \text { velocities of aircraft }(\mathrm{m} / \mathrm{s}) \\
& x_{\text {cpa }}, y_{\text {cpa }}=X \text { and } Y \text { components of closest point of approach }(\mathrm{m}) \\
& x_{m}, y_{m} \quad=X \text { and } Y \text { components of the maneuver taken by the ownship }(\mathrm{m}) \\
& x_{t}, y_{t}=X \text { and } Y \text { components of the turning maneuver }(\mathrm{m}) \\
& x_{l}, y_{l} \quad=X \text { and } Y \text { components of the linear segment of the avoidance maneuver } \\
& (\mathrm{m}) \\
& y_{\mathrm{cpa}}^{\chi_{t}}=Y \text { component of the closest point of approach assuming the course is } \\
& \text { equal to } \chi_{t}(\mathrm{~m}) \\
& z \quad=\text { change of variable for } \sin \theta_{\text {cpa }} \\
& z_{1}, z_{2}, z_{3}=\text { three roots of } z \\
& \delta_{a} \quad=\text { deflection of ailerons in the time domain (rad) } \\
& \delta_{r} \quad=\text { slack parameter } \\
& \delta T_{3} \quad=\text { total time during segment } 3 \text { where the bank angle is at a constant } \\
& \text { value of } \phi_{\max }(\mathrm{s}) \\
& \delta \chi_{1}, \delta \chi_{2}, \delta \chi_{3}, \delta \chi_{4}, \delta \chi_{5}=\text { changes in course during segments } 1,2,3,4 \text { and } 5 \text { (rad) } \\
& \delta \chi_{2,4}=\text { change in course during segments } 2 \text { and } 4 \text { combined (rad) } \\
& \theta_{\text {cpa }}=\text { angle between the forward path of the intruder and the closest point of } \\
& \text { approach of the ownship (rad) } \\
& \sigma \quad=\text { independent variable used for integration } \\
& \phi, \phi_{\max }=\text { bank angle and maximum bank angle (rad) } \\
& \phi_{1}, \phi_{2}, \phi_{3}, \phi_{4}, \phi_{5} \quad=\text { bank angles during segments } 1,2,3,4 \text { and } 5(\mathrm{rad}) \\
& \phi_{2,4}, \dot{\phi}_{2,4}=\text { bank angle and bank-angle rate during segments } 2 \text { and } 4 \text { combined } \\
& (\mathrm{rad}),(\mathrm{rad} / \mathrm{s})
\end{aligned}
$$




$\begin{array}{ll}\dot{\phi}_{\max } & =\text { maximum bank-angle rate }(\mathrm{rad} / \mathrm{s}) \\ \dot{\phi}_{1}, \dot{\phi}_{2}, \dot{\phi}_{3}, \dot{\phi}_{4}, \dot{\phi}_{5} & =\text { bank-angle rates during segments } 1,2,3,4 \text { and } 5(\mathrm{rad} / \mathrm{s}) \\ \phi^{f} & =\text { free response of the bank angle }(\mathrm{rad}) \\ \dot{\phi}, \ddot{\phi} & =\text { first and second time derivatives of the bank angle }(\mathrm{rad} / \mathrm{s}),\left(\mathrm{rad} / \mathrm{s}^{2}\right) \\ \chi & =\text { course of aircraft }(\mathrm{rad}) \\ \chi_{\mathrm{cpa}} & =\text { change in course during the turning maneuver }(\mathrm{rad}) \\ \chi_{t} & =\text { course rate and maximum course rate }(\mathrm{rad} / \mathrm{s}) \\ \dot{\chi}, \dot{\chi}_{\max } & \end{array}$

\section{Introduction}

The use of unmanned aircraft systems (UASs) in commercial and civil applications has been expanding rapidly in recent years. Many UAS missions will require simultaneous operation with existing airspace users. UASs currently have limited access to the National Airspace System (NAS) because they do not have the ability to detect and avoid other air traffic. Among many regulatory and technology issues, safety is the foremost concern and the most significant challenge to overcome before UAS integration into the NAS can be achieved. The Federal Aviation Administration, which is the national aviation authority in the United States, calls for a target level of safety that is more stringent than the see-and-avoid requirement for manned aircraft [1].

Robust and reliable detect-and-avoid (DAA) systems will be necessary for UASs to provide the required target level of safety. Typically, a complete functional detect-and-avoid system comprises of sensors and associated trackers, collision detection, risk assessment, collision-avoidance, and selfseparation algorithms.

The main role of the sensor and tracker is to detect any of the various types of hazards, such as traffic or terrain, and track the motion of the detected object to gain sufficient confidence that the detection is valid. Electro-optical and infrared cameras, light detection and ranging, and radar are examples of sensors employed to detect non-cooperative traffic [2]. Non-cooperative traffic means that data about potential conflicts are not communicated or transmitted to the ownship UAS from 
the conflicting intruders. The traffic alert and collision-avoidance system (TCAS) and automatic dependent surveillance-broadcast (ADS-B) are examples of systems for detecting cooperative intruders. Both cooperative and non-cooperative sensors can be used with DAA systems, and both can be used in conjunction with the results of this paper.

Not every aircraft that is observed by the detection system presents a risk of collision or violation of the well-clear boundary. The self-separation algorithm must therefore identify potential wellclear violations and plan new paths that remain well clear of intruder aircraft while optimizing an objective function or performance metric. Self-separation is designed to prevent future collisionavoidance maneuvers and is achieved using less-aggressive maneuvers. If the well-clear boundary is penetrated, the collision detection and collision-avoidance system must be used to detect potential collisions and compute collision-free paths. A collision avoidance maneuver is considered as the last resort effort to steer the UAS onto a safe course to prevent an imminent collision and may require an aggressive change in flight path.

The design of a DAA system for UASs should also address regulatory requirements, as well as performance and reliability standards. Initial efforts to address performance, design, construction, and reliability requirements of DAA systems for UASs were discussed in the white papers produced by Radio Telecommunications Corporation of America in RTCA SC-228 [1]. An excellent review of existing regulations, standards, and recommended practices, along with suggestions and recommendations for DAA requirements to facilitate the UAS integration into the NAS system, were discussed in Refs. [3-5]. Specific design parameters required by a DAA system, such as the sensor angular resolution, field of view, and minimum time and detection range, that are needed to prevent a collision assuming a two-dimensional (2-D) head-on encounter geometry, were addressed in Ref. [6]. Sensor and tracking requirements were derived for a radar-based DAA system considering 2-D flight worst-case encounter scenarios using exhaustive Monte Carlo simulations in Ref. [7]. The authors in Ref. [8] proposed a framework that consisted of a target level of safety approach using an event-tree format to develop specific DAA effectiveness standards linking UAS characteristics and operating environments to midair collision risk quantified by a fatality rate.

Among these requirements and design specifications, developing sensors that achieve sufficiently 
large target detection ranges for effective self-separation and collision avoidance is a crucial aspect of a viable DAA solution. This paper provides an exact numerical solution and a closed-form analytical approximation to the minimum detection range. An outline of the paper is as follows. In Section II, derivation of the minimum detection range (MDR) $d_{\mathrm{MDR}}$ is given along with the appropriate definitions and assumptions. An overview of two existing methods is given in Section II A, followed by the new methods in Section IIB. In Section III, results are presented, providing a comparison of the four methods: the two presented in this paper, and those presented in Ref.[6] and [9]. Finally, conclusions are presented in Section IV.

\section{Minimum Detection Range Formulation}

The minimum required detection range arises from the time required to complete the detection and avoidance of an intruder. The minimum time for the DAA system to be able to track the intruder, detect a collision or well-clear violation, plan an avoidance maneuver, wait for human review/approval, and fly the maneuver determines the distance at which the UAS must detect the intruder. The detection of a well-clear violation or collision threat must be accomplished at no less than the minimum detection range to allow the ownship to execute the maneuver with sufficient time so that the closest point of approach is greater than or equal to the separation requirement. A time sequence for the DAA system, similar to the proposed sequence in Ref. [6], is shown in Fig. 1. According to the time sequence, the time required for DAA operations, $t_{\mathrm{DAA}}$, is defined as the sum of the computation time, $t_{c}$, and the time that is required to maneuver, $t_{m}$.

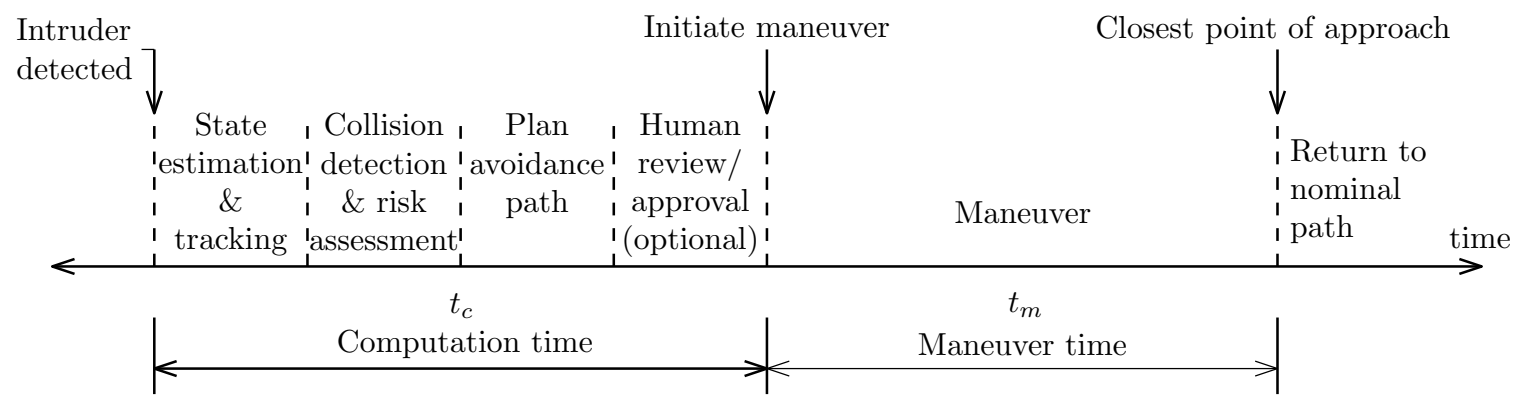

Fig. 1 Proposed timeline for the detect-and-avoid system [6].

Current manned aviation regulations have no explicit values for separation requirements; how- 
ever, various attempts have been made to define them. For collision avoidance of manned aviation, a common requirement is the near midair collision (NMAC) volume, which is a disk-shaped volume with a horizontal radius of $500 \mathrm{ft}$ and a vertical height of $200 \mathrm{ft}$ [10-12]. No such requirements exist for UASs; however, the NMAC volume could be used as a conservative requirement. For selfseparation, the well-clear boundary is more ambiguous and depends on the specific aircraft involved and their associated speeds and altitudes. Recent efforts to define this boundary for UASs have resulted in values in the range of 0.5 to $1 \mathrm{n}$ mile [13]. For UASs, the potential ownship and intruder aircraft can vary widely in vehicle size, weight, and airspeed; and the separation requirements could be scaled accordingly. For this work, we will assume a purely geometric safety volume centered around the aircraft as shown in Fig. 2. The general choice for this volume is a cylinder of radius $R_{s}$ and height $h_{s}$ centered at the current location of the aircraft. This volume will be used to represent the well-clear or NMAC volume. From this volume, a well-clear violation or an NMAC is defined as an incident that occurs when two aircraft pass with a distance less than $R_{s}$ horizontally and $h_{s} / 2$ vertically.

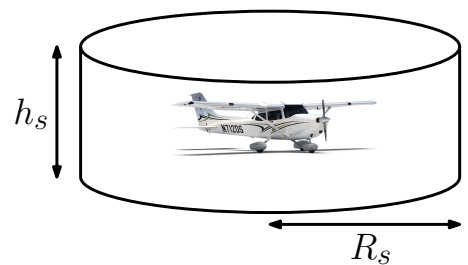

Fig. 2 General volume used to represent the well-clear or NMAC conditions.

Various types of sensors exist for detecting intruder aircraft, and they can be located at different locations, such as on the ownship aircraft, a stationary ground-based platform, or a moving groundbased platform. If the sensor is fixed to the ownship, the minimum detection range calculated in this paper can be directly used as the minimum sensing range requirement of the sensor. If the sensor is used with a ground-based system, the minimum detection range determines how close an aircraft can fly to the edge of maximum surveillance range and still be guaranteed to avoid intruders. The equations developed in this paper for the minimum detection range are independent of the specific sensor type chosen; however, because of field-of-view limits, multiple sensors may be required to achieve the desired field of view. 
The analysis in this paper assumes that there is only one intruder aircraft. If there is more than one intruder, then the avoidance maneuver will likely be more complex and will require a greater detection range. This paper considers the longest-detection-range encounter that occurs when both the ownship and intruder are flying at a constant altitude, course, and airspeed in a direct head-on approach. Various types of avoidance maneuvers can be taken by the ownship to avoid the intruder's safety volume. First, the ownship could perform a turning maneuver at a constant altitude to stay outside the horizontal safety radius $R_{s}$. Second, the ownship could perform a climbing/descending maneuver without turning to achieve a relative altitude equal to or greater than $h_{s} / 2$ while inside the safety radius. Third, the ownship could both turn and climb/descend simultaneously to avoid either the safety radius or the safety altitude: whichever comes first. Finally, the ownship could adjust its speed in addition to one of the previous maneuvers. Because $R_{s}$ is generally much larger than $h_{s}$, a constant-altitude turning maneuver will require the largest distance to avoid the safety volume and will be the focus of our avoidance-maneuver analysis. For simplicity, the ownship velocity is assumed to be constant and above the stall speed of the ownship. The speed of the ownship and intruder are defined as $v_{o}$ and $v_{i}$, respectively.

In this analysis, the ownship's turning dynamics follow the coordinated-turn relationships [14]

$$
\begin{gathered}
\dot{\chi}=\frac{g}{v_{o}} \tan \phi \\
R_{\min }=\frac{v_{o}}{\dot{\chi}_{\max }}=\frac{v_{o}^{2}}{g \tan \phi_{\max }},
\end{gathered}
$$

where $\dot{\chi}$ is the course rate, $\phi$ is the bank angle, $R_{\min }$ is the minimum turning radius, $\dot{\chi}_{\max }$ is the maximum course rate, $\phi_{\max }$ is the maximum bank angle, and $g$ is the gravitational constant. This analysis is intended for use with fixed-wing aircraft with turning dynamics that are well modeled by the coordinated-turn relationships. The analysis is also valid for other types of aircraft, such as rotorcraft, provided their turn dynamics are approximated by the coordinated turn.

The assumptions that have been made and other real-world issues may limit the validity of the analysis and, as a result, additional range may be required in practice. Ownship and intruder states are not known perfectly due to state estimation errors resulting from imperfect inertial measurement unit, Global Positioning System, and detection sensors. The intruder may maneuver during the 
encounter by turning, changing altitude, or changing speed. Finally, the ownship may not follow the ideal coordinated-turn flight trajectory. Each of these issues will need to be considered before final sensor requirements can be made; however, the results of this paper provide a solid foundation to build upon.

\section{A. Prior Approaches}

One method, known as the tactical separation assisted flight environment (TSAFE) resolution algorithm (developed by Erzberger [15]), deserves mention. The TSAFE resolution algorithm does not solve for the minimum detection range specifically; however, various supplemental equations are common between our method and the TSAFE resolution algorithm, including the position and course dynamics of the ownship. In the TSAFE resolution algorithm, two aircraft are given arbitrary positions, headings, and speeds relative to each other. Using these initial conditions, resolution maneuvers are calculated based on one or both of the aircraft performing a horizontal turning maneuver followed by straight-line flight. The turning maneuvers are performed by rightor left-turn maneuvers, with the bank angle and turn time as the control variables. By varying each of the variables, equations are developed to calculate the closest point of approach (CPA) between the two aircraft during the turning maneuver and during the straight-line flight. The final CPA between the two aircraft is then found by taking the minimum of the CPA during the turning maneuver and the straight-line portion. The last step of the TSAFE resolution algorithm is to choose the appropriate bank angle, turn direction, and turn time, which result in the CPA being greater than the required safe distance. Although the TSAFE resolution algorithm was not formulated to find the minimum detection range, the CPA equations used by TSAFE resolution could be used in an iterative manner to solve for the minimum detection range by adjusting the starting distance between the two aircraft until the CPA equals the required safe distance.

Two approaches developed specifically for calculating minimum detection range are found in the literature. One approach, developed by Geyer et al. [6], is referred to in this paper as the turntime (TT) approach. The second approach, developed by Sahawneh et al. [9], is referred to as the geometric-tangent (GT) approach. Both approaches assume an instantaneous bank-angle maneuver 
and a head-on, constant-altitude, constant-velocity encounter. A brief description of these two methods is given in the following subsections.

\section{Turn Time Approach}

Using the TT method proposed in Ref. [6], the minimum detection range is calculated using the expression

$$
d_{\mathrm{MDR}}=\left(v_{o}+v_{i}\right)\left(t_{c}+t_{t}\right)
$$

where $t_{t}$ is the time when the ownship and intruder are closest as the ownship is executing a turning maneuver. The turn time is found using equations for the north and east positions of both the ownship and intruder as functions of time, as well as the assumption that the turn time is approximately equal to the time to collision in the absence of a maneuver. Using this time-based approach, the minimum detection range is expressed as

$$
d_{\mathrm{MDR}} \approx\left(v_{o}+v_{i}\right)\left(t_{c}+\sqrt{\frac{2 R_{s} \cot \phi_{\max }}{g}}\right) .
$$

In Ref. [6], the authors acknowledged that their solution was an approximation to the true minimum detection range, and they stated that it was meant to be used as a heuristic for choosing the right sensor and its resolution. They further stated that it was not suitable for small distances and velocities, but they did not specifically define limiting values.

\section{Geometric Tangent Approach}

The GT method proposed in Ref. [9] approximates the minimum detection range as

$$
d_{\mathrm{MDR}}=\left(v_{o}+v_{i}\right) t_{c}+d_{m}
$$

where $d_{m}$ is the distance between the ownship and the intruder when the ownship starts maneuvering. In this method, the ownship executes a turning maneuver with a constant turning radius. The closest point of approach is then assumed to occur when the ownship is located on the edge of the safety circle around the intruder and the turning radius of the ownship is tangent to the safety circle around the intruder. Using this geometric relationship, the minimum detection range is expressed 


$$
d_{\mathrm{MDR}} \approx\left(v_{o}+v_{i}\right) t_{c}+\sqrt{R_{s}^{2}+2 R_{s} \frac{v_{o}^{2}}{g \tan \phi_{\max }}}+\frac{v_{i} v_{o}}{g \tan \phi_{\max }} \cos ^{-1}\left(\frac{v_{o}^{2}}{v_{o}^{2}+R_{s} g \tan \phi_{\max }}\right) .
$$

In Ref. [9], the authors acknowledged that their solution was an under-approximation and stated that compensation could be made by selecting a positive slack parameter, $\delta_{r}$, to obtain $\bar{d}_{\mathrm{MDR}}=\left(1+\delta_{r}\right) d_{\mathrm{MDR}}$. Slack parameters must be found using experimental results, such as those from Monte Carlo simulations, but insights into appropriate slack parameter values were not given.

\section{B. New Approaches}

The TT and GT approaches both make the assumption that the ownship executes a turning maneuver by instantaneously banking to a specified angle. This assumption simplifies the derivation of the minimum detection range by confining the trajectory of the ownship to a circular arc. In the turning maneuver of real aircraft, an instantaneous bank-angle maneuver is not physically possible. Instead, the bank angle has a transient response resulting from the deflection of the ailerons. The response of the bank angle determines the course rate of a coordinated turn as shown in Eq. (1). This non-constant course rate results in a trajectory that is not circular and must be determined by numerical integration of complex time-based functions. Considering the bank-angle dynamics results in a more accurate prediction of the minimum detection range that is larger than the predictions of the TT and GT methods. The proposed method that takes into account the bank-angle dynamics is called the time-based geometric velocity vectors (TGVVs) approach and is described fully in this paper.

The second method presented in this paper, known as the geometric velocity vectors (GVVs) approach, is a special case of the TGVV approach that maintains the assumption of an instantaneous bank-angle maneuver. By using this assumption, the GVV method allows flight trajectories to be represented geometrically as circular paths instead of functions of time, which allows a closed-form analytical solution for the minimum detection range to be derived. We will show under what conditions, the instantaneous bank-angle assumption is valid, allowing the GVV method to be used with confidence. We will further show that the GVV method produces more accurate approximations to the TGVV solution over a wider range of conditions than the solutions offered by the TT and GT 
approaches. The TGVV and GVV methods also allow the turning angle for the avoidance maneuver to be defined by the analyst instead of prescribing a $90 \mathrm{deg}$ turn. Although this may result in a slightly larger $d_{\mathrm{MDR}}$, it also allows deviations from the nominal flight path to be reduced at the discretion of the analyst.

\section{Problem Formulation}

A diagram for the total minimum detection range, $d_{\mathrm{MDR}}$, is shown in Fig. 3, and the resulting general equation for $d_{\mathrm{MDR}}$ is represented as

$$
d_{\mathrm{MDR}}=d_{o}+d_{i}+d_{\mathrm{CPA}},
$$

where $d_{o}$ is the total head-on distance traveled by the ownship, and $d_{i}$ is the total head-on distance traveled by the intruder. The final term $d_{\mathrm{CPA}}$ is the remaining head-on distance between the ownship and intruder when the closest point of approach has been reached.

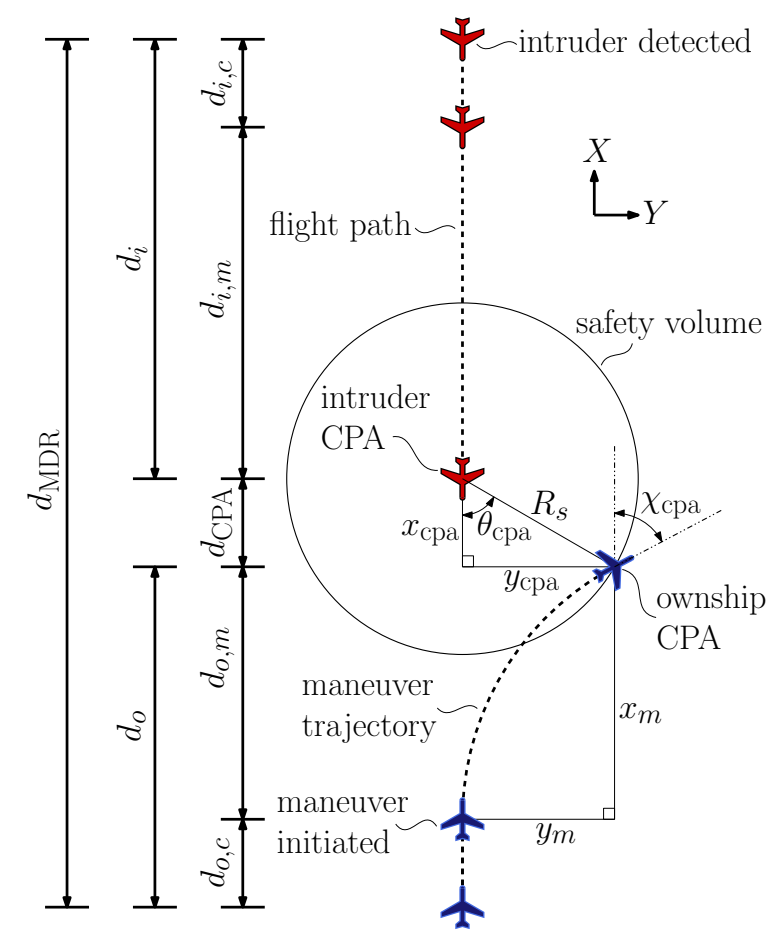

Fig. 3 The total minimum detection range, $d_{\mathrm{MDR}}$, needed. A representation of the CPA.

In Fig. 3, we can also see that the variables $d_{o}$ and $d_{i}$ can both be broken down into two subsegments each. The total head-on distance traveled by the ownship can be defined as $d_{o}=$ 
$d_{o, c}+d_{o, m}$, where $d_{o, c}$ is the head-on distance traveled by the ownship during computation time, and $d_{o, m}$ is the head-on distance traveled by the ownship while it is executing its maneuver. Similarly, the total head-on distance traveled by the intruder can be defined as $d_{i}=d_{i, c}+d_{i, m}$, where $d_{i, c}$ is the head-on distance traveled by the intruder during computation time, and $d_{i, m}$ is the head-on distance traveled by the intruder while the ownship is executing its maneuver. Using these definitions, the minimum detection range becomes

$$
d_{\mathrm{MDR}}=d_{o, c}+d_{o, m}+d_{i, c}+d_{i, m}+d_{\mathrm{CPA}}
$$

When analyzing the interaction between two aircraft during an avoidance maneuver, the CPA is a significant point of interest and a detailed derivation of this location is the basis upon which the new methods are built. The previous GT approach also uses the CPA as the basis of their derivation of the minimum detection range; however, the GT approach differs in where the CPA is located. In the GT method, the CPA is assumed to be located where the circular arc that is transversed by the ownship during its avoidance maneuver becomes tangent to the safety circle drawn around the intruder. In the TGVV and GVV methods, the CPA is also located on the edge of the safety circle around the intruder; however, the tangency assumption is removed. Instead, the ownship is assigned an arbitrary course angle $\chi_{\text {cpa }}$ when it is located on the edge of the safety circle, as seen in Fig. 3. This illustration is represented in a right-handed $X-Y-Z$ inertial reference frame, and the variable $\chi_{\mathrm{cpa}}$ is defined relative to the $X$ axis. In addition to $\chi_{\mathrm{cpa}}$, an additional angle is needed to define the location of the ownship on the edge of the safety circle when the CPA is reached, which is shown by the variable $\theta_{\text {cpa }}$ in Fig. 3. The variable $\theta_{\text {cpa }}$ is measured relative to the negative $X$ axis and represents the angle between the forward flight path of the intruder and the line connecting the CPA of the intruder and ownship. Using the definition of $\theta_{\text {cpa }}$, a right triangle is formed with its hypotenuse equal to the safety radius $R_{s}$ and the two sides equal to $x_{\text {cpa }}$, and $y_{\text {cpa }}$.

The CPA can be identified by taking the derivative of the range with respect to time, known as the range rate, and setting it to zero. The range rate is calculated as

$$
\dot{r}=\frac{\mathbf{p}_{o}-\mathbf{p}_{i}}{\left\|\mathbf{p}_{o}-\mathbf{p}_{i}\right\|} \cdot\left(\mathbf{v}_{o}-\mathbf{v}_{i}\right)
$$

where $\mathbf{p}_{o}, \mathbf{p}_{i}, \mathbf{v}_{o}$, and $\mathbf{v}_{i}$ are vectors defined in the inertial reference frame. The vector $\mathbf{p}_{o}$ is the 
position vector of the ownship, $\mathbf{p}_{i}$ is the position vector of the intruder, $\mathbf{v}_{o}$ is the velocity vector of the ownship, and $\mathbf{v}_{i}$ is the velocity vector of the intruder. Using Fig. 3, the position and velocity vectors can be defined in terms of the safety radius $R_{s}$, the ownship and intruder velocities $v_{o}$, and $v_{i}$; and the unknown variables $x_{\mathrm{cpa}}, y_{\mathrm{cpa}}$, and $\chi_{\mathrm{cpa}}$. Because the aircraft are assumed to be flying at constant altitude, the $\mathrm{Z}$ component of the position and velocity vectors will be neglected. The origin is defined as the intruder position when the CPA is reached so that $\mathbf{p}_{i}=(0,0)$. Using the intruder's position as the origin, the ownship's position is then defined as $\mathbf{p}_{o}=\left(-x_{\mathrm{cpa}}, y_{\mathrm{cpa}}\right)$. The intruder's velocity vector is defined to be in the $-X$ direction; therefore, the intruder's velocity vector is defined as $\mathbf{v}_{i}=\left(-v_{i}, 0\right)$. The ownship's velocity vector points in the direction of $\chi_{\text {cpa }}$ and is defined by $\mathbf{v}_{o}=\left(v_{o} \cos \chi_{\text {cpa }}, v_{o} \sin \chi_{\text {cpa }}\right)$. Substituting these position and velocity vectors into Eq. (6) and equating it to zero results in

$$
\begin{aligned}
0 & =\frac{\left(-x_{\mathrm{cpa}}, y_{\mathrm{cpa}}\right)-(0,0)}{\left\|\left(-x_{\mathrm{cpa}}, y_{\mathrm{cpa}}\right)-(0,0)\right\|} \cdot\left(\left(v_{o} \cos \chi_{\mathrm{cpa}}, v_{o} \sin \chi_{\mathrm{cpa}}\right)-\left(-v_{i}, 0\right)\right), \\
& =\frac{1}{R_{s}}\left[v_{o} \sin \chi_{\mathrm{cpa}} y_{\mathrm{cpa}}-\left(v_{i}+v_{o} \cos \chi_{\mathrm{cpa}}\right) x_{\mathrm{cpa}}\right]
\end{aligned}
$$

where we now have a single equation with unknown variables $x_{\mathrm{cpa}}, y_{\mathrm{cpa}}$, and $\chi_{\mathrm{cpa}}$.

If an instantaneous bank-angle maneuver is assumed, as in the GVV method, the three unknown variables can be expressed in terms of the single unknown variable $\theta_{\text {cpa }}$. The resulting equation can then be solved for $\theta_{\mathrm{cpa}}$ explicitly, from which the specific values for $x_{\mathrm{cpa}}, y_{\mathrm{cpa}}$, and $\chi_{\mathrm{cpa}}$ are found.

If on the other hand, a non-instantaneous bank-angle maneuver is assumed; as in the TGVV method, the variable $\chi_{\mathrm{cpa}}$ cannot be expressed in terms of $\theta_{\mathrm{cpa}}$. For this case, $\chi_{\mathrm{cpa}}$ is found by integration of the turning dynamics of the aircraft over time. Because the variable $\chi_{\text {cpa }}$ is now based on time, the three unknown variables are expressed in terms of the time to maneuver to the CPA, $t_{m}$, instead of the unknown variable $\theta_{\text {cpa }}$. After substituting these expressions back into Eq. (7), the resulting equation cannot be solved for $t_{m}$ explicitly and must instead be found through numerical methods. Once $t_{m}$ has been determined, the specific values for $x_{\text {cpa }}, y_{\text {cpa }}$, and $\chi_{\text {cpa }}$ can be found.

Using Fig. 3 and the definitions of the CPA as described previously, $d_{\mathrm{CPA}}$ is equal to $x_{\mathrm{cpa}}$. From this figure, we can also see that $d_{o, m}$ is equal to the $X$ component of the maneuvering ownship 
when it reaches the CPA, $x_{m}$. The head-on distance traveled by the intruder while the ownship is executing its maneuver is a linear function of the time it takes the ownship to maneuver to the CPA as $d_{i, m}=v_{i} t_{m}$. Finally, because the ownship and intruder are assumed to be traveling at constant velocity, $d_{o, c}$ and $d_{i, c}$ are linear functions of the computation time and are expressed as $d_{o, c}=v_{o} t_{c}$ and $d_{i, c}=v_{i} t_{c}$, respectively. With each of these definitions, the general equation for the minimum detection range finally becomes

$$
d_{\mathrm{MDR}}=\left(v_{o}+v_{i}\right) t_{c}+x_{m}+v_{i} t_{m}+x_{\mathrm{cpa}} .
$$

The remaining variables $x_{m}, t_{m}$, and $x_{\mathrm{cpa}}$ are dependent on the specific maneuver taken by the ownship and the location on the edge of the safety circle where the CPA occurs. The specific maneuver taken by the ownship and the resulting location of the CPA differ between the TGVV and GVV methods and will be defined in the following two sections along with the resulting minimum detection range.

\section{Time-Based Geometric Velocity Vectors Approach}

As stated previously, the TGVV approach assumes that the turning maneuver executed by the ownship is driven by a non-instantaneous bank angle change and the resulting trajectory must be characterized by numerical integration of time-based turn dynamics. The first step in characterizing the trajectory is to define the $X$ and $Y$ positions of the ownship during its maneuver as functions of time as

$$
\begin{aligned}
& p_{x}(t)=\int_{t_{0}}^{t} v_{x}(\sigma) d \sigma=\int_{t_{0}}^{t} v_{o} \cos \chi(\sigma) d \sigma \\
& p_{y}(t)=\int_{t_{0}}^{t} v_{y}(\sigma) d \sigma=\int_{t_{0}}^{t} v_{o} \sin \chi(\sigma) d \sigma .
\end{aligned}
$$

These positions are measured relative to the location where the ownship initiates its avoidance maneuver and the time is measured relative to the time when the ownship initiates its avoidance maneuver, $t_{0}$. The variables $v_{x}$ and $v_{y}$ are the $X$ and $Y$ velocity components of the ownship as functions of time while it performs its turning maneuver. The variable $\chi$ is the course of the ownship as a function of time while it performs its turning maneuver, and $\sigma$ is the independent variable of integration. 
Assuming the ownship performs a coordinated-turn maneuver, the course of the aircraft can be found by integrating the course rate from Eq. (1) as

$$
\chi(t)=\int_{t_{0}}^{t} \dot{\chi}(\sigma) d \sigma=\int_{t_{0}}^{t} \frac{g}{v_{o}} \tan \phi(\sigma) d \sigma .
$$

For the GVV, method which will be described later, $\phi$ is assumed to be a step function with a magnitude of $\phi_{\max }$; however, the TGVV approach assumes that the turning maneuver executed by the ownship is influenced by the bank-angle dynamics. At this point, any desired banking dynamics could be used to define the course as a function of time; however, we have chosen to use a first-order transfer function that describes the roll rate of the aircraft, $p$, in response to the deflection of the ailerons $\delta_{a}$. The roll rate is integrated to get the bank angle. A block diagram of this system is shown in Fig. 4, where $K$ and $\tau$ are general first-order system parameters, and $s$ is the Laplace variable.

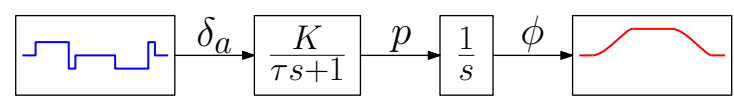

Fig. 4 Block diagram of the bank-angle response due to aileron inputs.

Using this dynamic model, we design a set of aileron commands to achieve a desired bank-angle response as shown in Fig. 5(a). In interval 1, a positive aileron step is used at $t_{0}$ to achieve a constant bank-angle rate, $\dot{\phi}_{\max }$. In interval 2 , a negative aileron step is used at $t_{1}$ to stop the rolling motion at the maximum bank angle, $\phi_{\max }$. In interval 3 , the aircraft holds this constant bank angle, beginning at $t_{2}$, until it is time to return to level flight. The time at which the aircraft needs to begin returning to level flight is shown by $t_{3}$ and is chosen so that the total change in course resulting from the turning maneuver is equal to $\chi_{t}$. In interval 4 , a negative aileron step is used at $t_{3}$ to achieve a constant bank-angle rate, $-\dot{\phi}_{\max }$. In interval 5 , a positive aileron step is used at $t_{4}$ to stop the rolling motion with the bank-angle returns at zero. Finally, at $t_{5}$, the aircraft remains in level flight at the desired course, $\chi_{t}$, for the remainder of the avoidance maneuver. In some cases, the aircraft may not reach the maximum bank angle before it is time to return to level flight to ensure the proper $\chi_{t}$ is achieved. In these cases, the bank angle has the response shown in Fig. 5(b), from which we notice $t_{2}$ and $t_{3}$ occur at the same time instant and there is no longer an interval 3 . We 
also notice that intervals 2 and 4 are combined in terms of the negative step input to the system from the ailerons.

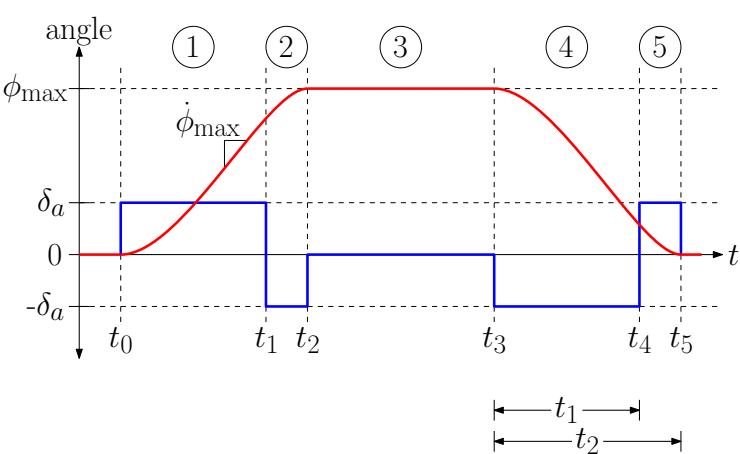

(a) Case A: $\phi_{\max }$ is reached.

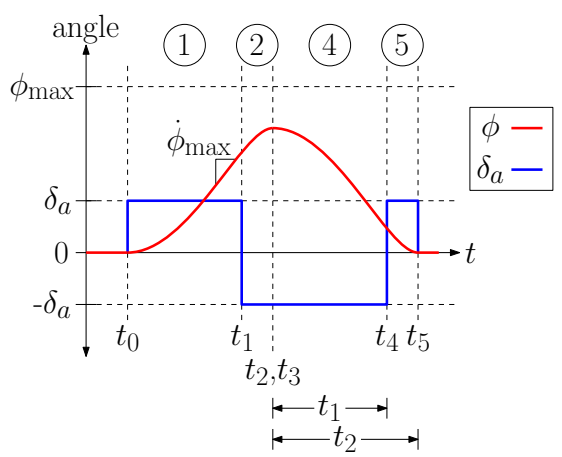

(b) Case B: $\phi_{\max }$ is not reached.

Fig. 5 Bank-angle response to aileron step inputs.

From Fig. 5, the segments of the bank-angle response where $\phi$ is changing as a result of the aileron input are intervals $1,2,4$, and 5 . The time response of $\phi$ to step inputs in the aileron command can be derived from the ordinary differential equation describing the bank-angle dynamics:

$$
\tau \ddot{\phi}+\dot{\phi}=K \delta_{a}(t)
$$

The bank-angle response due to an aileron step input of magnitude $\delta_{a}$ occurring at time $t_{0}$ is given by

$$
\phi(t)=K \delta_{a}\left[\tau e^{-\left(t-t_{0}\right) / \tau}-\tau+\left(t-t_{0}\right)\right]+\tau \dot{\phi}\left(t_{0}\right)\left(1-e^{-\left(t-t_{0}\right) / \tau}\right)+\phi\left(t_{0}\right),
$$

whereas the bank-rate response is given by

$$
\dot{\phi}(t)=K \delta_{a}\left(1-e^{-\left(t-t_{0}\right) / \tau}\right)+\dot{\phi}\left(t_{0}\right) e^{-\left(t-t_{0}\right) / \tau} .
$$

The magnitude of the aileron step input is chosen so that the steady-state bank-angle rate is the prescribed maximum $\dot{\phi}_{\max }$, which gives

$$
\delta_{a}=\frac{\dot{\phi}_{\max }}{K}
$$

Substituting this value of $\delta_{a}$ back into the equations for $\phi(t)$ and $\dot{\phi}(t)$ results in equations in terms of $\tau$ and $\dot{\phi}_{\max }$ as

$$
\begin{aligned}
& \phi(t)=\tau \dot{\phi}_{\max }\left(e^{-\left(t-t_{0}\right) / \tau}-1\right)+\dot{\phi}_{\max }\left(t-t_{0}\right)+\tau \dot{\phi}\left(t_{0}\right)\left(1-e^{-\left(t-t_{0}\right) / \tau}\right)+\phi\left(t_{0}\right), \\
& \dot{\phi}(t)=\dot{\phi}_{\max }\left(1-e^{-\left(t-t_{0}\right) / \tau}\right)+\dot{\phi}\left(t_{0}\right) e^{-\left(t-t_{0}\right) / \tau}
\end{aligned}
$$


These equations are the general equations used to define the response of the bank angle to a positive or negative step input from the ailerons for cases A and B. The specific bank-angle responses for each interval are derived for both cases in the Appendix.

Tables 1 and 2 provide a summary of the specific bank-angle response for each interval, shown by $\phi_{i}$, for case $\mathrm{A}$ and case $\mathrm{B}$, respectively. Additionally, the transition times for each segment are shown by $t_{i}$, and the change in course during each segment is shown by $\delta \chi_{i}$. To determine if case A or case B is required for the bank-angle dynamics of the ownship, we use the value calculated for $\delta \chi_{3}$. If $\delta \chi_{3} \geq 0$, then case $\mathrm{A}$ is used; and if $\delta \chi_{3}<0$, then case $\mathrm{B}$ is used.

\begin{tabular}{|c|c|c|}
\hline Interval & \multicolumn{2}{|l|}{$\phi_{i}(t)$} \\
\hline 1 & \multicolumn{2}{|l|}{$\phi_{1}(t)=\tau \dot{\phi}_{\max }\left(e^{-t / \tau}-1\right)+\dot{\phi}_{\max } t$} \\
\hline 2 & \multicolumn{2}{|c|}{$\phi_{2}(t)=\tau \dot{\phi}_{\max }\left(1-2 e^{-\left(t-t_{1}\right) / \tau}+e^{-t / \tau}\right)-\dot{\phi}_{\max }\left(t-2 t_{1}\right)$} \\
\hline 3 & \multicolumn{2}{|l|}{$\phi_{3}(t)=\phi_{\max }$} \\
\hline 4 & \multicolumn{2}{|c|}{$\phi_{4}(t)=\tau \dot{\phi}_{\max }\left(1-e^{-\left(t-t_{3}\right) / \tau}\right)-\dot{\phi}_{\max }\left(t-t_{3}\right)+\phi_{\max }$} \\
\hline 5 & \multicolumn{2}{|c|}{$\phi_{5}(t)=\tau \dot{\phi}_{\max }\left(2 e^{-\left(t-t_{4}\right) / \tau}-1-e^{-\left(t-t_{3}\right) / \tau}\right)+\dot{\phi}_{\max }\left(t-2 t_{4}+t_{3}\right)+\phi_{\max }$} \\
\hline Interval & $t_{i}$ & $\delta \chi_{i}$ \\
\hline 1 & $t_{1}=-\tau \ln \left[\frac{e^{-\left(\phi_{\max } / \dot{\phi}_{\max }\right) / \tau}}{1+\sqrt{1-e^{-\left(\phi_{\max } / \dot{\phi}_{\max }\right) / \tau}}}\right]$ & $\delta \chi_{1}=\int_{t_{0}}^{t_{1}} \frac{g}{v_{o}} \tan \left(\phi_{1}(t)\right) d t$ \\
\hline 2 & $t_{2}=2 t_{1}-\frac{\phi_{\max }}{\phi_{\max }}$ & $\delta \chi_{2}=\int_{t_{1}}^{t_{2}} \frac{g}{v_{o}} \tan \left(\phi_{2}(t)\right) d t$ \\
\hline 3 & $t_{3}=t_{2}+\frac{R_{\min \delta \times 3}}{v_{o}}$ & $\delta \chi_{3}=\chi_{t}-\left(\delta \chi_{1}+\delta \chi_{2}+\left.\delta \chi_{4}\right|_{t_{3}=0, t_{4}=t_{1}}+\left.\delta \chi_{5}\right|_{t_{3}=0, t_{4}=t_{1}, t_{5}=t_{2}}\right.$ \\
\hline 4 & $t_{4}=t_{3}+t_{1}$ & $\delta \chi_{4}=\int_{t_{3}}^{t_{4}} \frac{g}{v_{o}} \tan \left(\phi_{4}(t)\right) d t$ \\
\hline 5 & $t_{5}=t_{3}+t_{2}$ & $\delta \chi_{5}=\int_{t_{4}}^{t_{5}} \frac{g}{v_{o}} \tan \left(\phi_{5}(t)\right) d t$ \\
\hline
\end{tabular}

Table 1 Bank angle, time intervals, and change in course for each segment of case A. 


\begin{tabular}{|c|c|c|}
\hline Interval & $\phi_{i}(t)$ & \\
\hline 1 & $\phi_{1}(t)=\tau \dot{\phi}_{\max }\left(e^{-t / \tau}-1\right)+\dot{\phi}_{\max } t$ & \\
\hline 2,4 & $\phi_{2,4}(t)=\tau \dot{\phi}_{\max }\left(1-2 e^{-\left(t-t_{1}\right) / \tau}+e\right.$ & $-t / \tau)-\dot{\phi}_{\max }\left(t-2 t_{1}\right)$ \\
\hline 5 & $\phi_{5}(t)=\tau \dot{\phi}_{\max }\left(e^{-t / \tau}\left(2 e^{t_{1} / \tau}-1\right)^{2}-\right.$ & $\left.1-2 \ln \left[2 e^{t_{1} / \tau}-1\right]\right)+\dot{\phi}_{\max } t$ \\
\hline Interval & $t_{i}$ & $\delta \chi_{i}$ \\
\hline 1 & numerical method in Appendix A 2 & $\delta \chi_{1}=\int_{t_{0}}^{t_{1}} \frac{g}{v_{o}} \tan \left(\phi_{1}(t)\right) d t$ \\
\hline 2,4 & $t_{2}=\tau \ln \left[2 e^{t_{1} / \tau}-1\right], t_{4}=t_{2}+t_{1}$ & $\delta \chi_{2,4}=\int_{t_{1}}^{t_{4}} \frac{g}{v_{o}} \tan \left(\phi_{2,4}(t)\right) d t$ \\
\hline 5 & $t_{5}=2 t_{2}$ & $\delta \chi_{5}=\int_{t_{4}}^{t_{5}} \frac{g}{v_{o}} \tan \left(\phi_{5}(t)\right) d t$ \\
\hline
\end{tabular}

Table 2 Bank angle, time intervals, and change in course for each segment of case B.

Using the information from Table 1, we create an expression for the course of the ownship after it initiates the avoidance maneuver for Case A as

$$
\chi(t)= \begin{cases}0 & \text { if } t \leq t_{0}, \\ \int_{t_{0}}^{t} \frac{g}{v_{o}} \tan \left(\phi_{1}(\sigma)\right) d \sigma & \text { if } t_{0}<t \leq t_{1}, \\ \delta \chi_{1}+\int_{t_{1}}^{t} \frac{g}{v_{o}} \tan \left(\phi_{2}(\sigma)\right) d \sigma & \text { if } t_{1}<t \leq t_{2}, \\ \delta \chi_{1}+\delta \chi_{2}+\int_{t_{2}}^{t} \frac{g}{v_{o}} \tan \left(\phi_{3}(\sigma)\right) d \sigma & \text { if } t_{2}<t \leq t_{3}, \\ \delta \chi_{1}+\delta \chi_{2}+\delta \chi_{3}+\int_{t_{3}}^{t} \frac{g}{v_{o}} \tan \left(\phi_{4}(\sigma)\right) d \sigma & \text { if } t_{3}<t \leq t_{4}, \\ \delta \chi_{1}+\delta \chi_{2}+\delta \chi_{3}+\delta \chi_{4}+\int_{t_{4}}^{t} \frac{g}{v_{o}} \tan \left(\phi_{5}(\sigma)\right) d \sigma & \text { if } t_{4}<t \leq t_{5}, \\ \chi_{t} & \text { if } t_{5}<t,\end{cases}
$$

Similarly, using the information from Table 2, we create an expression for the course of the ownship after it initiates the avoidance maneuver for Case B as 


$$
\chi(t)= \begin{cases}0, & \text { if } t \leq t_{0} \\ \int_{t_{0}}^{t} \frac{g}{v_{o}} \tan \left(\phi_{1}(\sigma)\right) d \sigma, & \text { if } t_{0}<t \leq t_{1} \\ \delta \chi_{1}+\int_{t_{1}}^{t} \frac{g}{v_{o}} \tan \left(\phi_{2,4}(\sigma)\right) d \sigma, & \text { if } t_{1}<t \leq t_{4} \\ \delta \chi_{1}+\delta \chi_{2,4}+\int_{t_{4}}^{t} \frac{g}{v_{o}} \tan \left(\phi_{5}(\sigma)\right) d \sigma, & \text { if } t_{4}<t \leq t_{5} \\ \chi_{t}, & \text { if } t_{5}<t\end{cases}
$$

Using Eqs. (15) and (16) for the course of the ownship and Eqs. (9) and (10) for the position of the ownship, the trajectory of the ownship is fully defined. Using this trajectory, we return to the analysis of the CPA location. The time to maneuver is the time it takes the ownship to initiate a turning maneuver until it reaches the CPA location on the edge of the safety circle around the intruder. This segment of the flight path of the ownship is shown in Fig. 3 and is composed of $X$ and $Y$ components: $x_{m}$ and $y_{m}$, respectively. These $X$ and $Y$ components can be defined in terms of the equations for $p_{x}(t)$ and $p_{y}(t)$, Eqs. (9) and (10), and the time to maneuver as

$$
\begin{aligned}
& x_{m}=p_{x}\left(t_{m}\right), \\
& y_{m}=p_{y}\left(t_{m}\right),
\end{aligned}
$$

From Eq. (7), we have three unknown variables $x_{\mathrm{cpa}}, y_{\mathrm{cpa}}$, and $\chi_{\mathrm{cpa}}$. Using Fig. 3 , we see that the unknown variable $y_{\text {cpa }}$, which defines the $Y$ component of the CPA, has the same value as the $Y$ component of the maneuvering ownship $y_{m}$ at the CPA. Accordingly, $x_{\text {cpa }}$ and $y_{\text {cpa }}$ can be expressed as

$$
\begin{aligned}
& y_{\mathrm{cpa}}=y_{m}=p_{y}\left(t_{m}\right), \\
& x_{\mathrm{cpa}}=\sqrt{R_{s}^{2}-y_{\mathrm{cpa}}^{2}}=\sqrt{R_{s}^{2}-p_{y}\left(t_{m}\right)^{2}},
\end{aligned}
$$

Similarly, $\chi_{\text {cpa }}$ can be expressed as

$$
\chi_{\mathrm{cpa}}=\chi\left(t_{m}\right) .
$$

After substituting these expressions for $x_{\mathrm{cpa}}, y_{\mathrm{cpa}}$, and $\chi_{\mathrm{cpa}}$ into Eq. (7) and simplifying, we get

$$
0=p_{y}\left(t_{m}\right) v_{o} \sin \chi\left(t_{m}\right)-\sqrt{R_{s}^{2}-p_{y}\left(t_{m}\right)^{2}}\left(v_{i}+v_{o} \cos \chi\left(t_{m}\right)\right)
$$


This equation is now a function of a single variable $t_{m}$, which can be solved for using the NewtonRaphson method. Once the value of $t_{m}$ has been found, the three variables $x_{\text {cpa }}, y_{\text {cpa }}$, and $\chi_{\text {cpa }}$ can then be calculated, along with the variables $x_{m}$ and $y_{m}$.

Now that the CPA location has been defined, we return to the calculation of the minimum detection range shown in Eq. (8). With the values for $x_{m}, t_{m}$, and $x_{\text {cpa }}$ just derived, the minimum detection range can be defined as

$$
d_{\mathrm{MDR}}=\left(v_{o}+v_{i}\right) t_{c}+p_{x}\left(t_{m}\right)+v_{i} t_{m}+\sqrt{R_{s}^{2}-p_{y}\left(t_{m}\right)^{2}}
$$

where $t_{m}$ is found from Eq. (22) using the Newton-Raphson method; $p_{x}\left(t_{m}\right)$ and $p_{y}\left(t_{m}\right)$ are found from Eqs. (9) and (10); and $\chi\left(t_{m}\right)$ is generally defined in Eq. (11), but is specifically defined for both case A and case B in Eqs. (15) and (16), respectively.

\section{Geometric Velocity Vectors Approach}

The TGVV method described previously incorporates the bank-angle dynamics of the ownship into the avoidance path. If the bank-angle dynamics of the ownship are fast relative to the maneuver time, then the assumption of an instantaneous bank angle becomes more realistic. For the GVV method, we make this assumption; in the results section (Sec. III), we show under what conditions this assumption is valid by comparing it to the TGVV method.

Instead of solving the bank-angle dynamics, we begin by defining the geometry that can be used to calculate the minimum detection range as shown in Fig. 6. From this figure, we see that there are two different geometrical cases that can occur while the ownship is maneuvering. For the first case, shown in Fig. 6(a), the ownship turns with a small turning radius as compared to the safety radius and is able to complete its turning maneuver before reaching the CPA location. This means that, after the ownship completes its turning maneuver, it will fly straight until the CPA location is reached. The $X$ and $Y$ components of the turning segment of the maneuver are represented by the variables $x_{t}$ and $y_{t}$, and the $X$ and $Y$ components of the linear segment of the maneuver are represented by $x_{l}$ and $y_{l}$. In the second case, shown in Fig. 6(b), the ownship turns gradually and reaches the CPA location before completing its turning maneuver. This means that the ownship will still be in a banked turn as it passes the CPA location. The $X$ and $Y$ components of the turning 
segment of the maneuver for this case are similarly represented by $x_{t}$ and $y_{t}$.

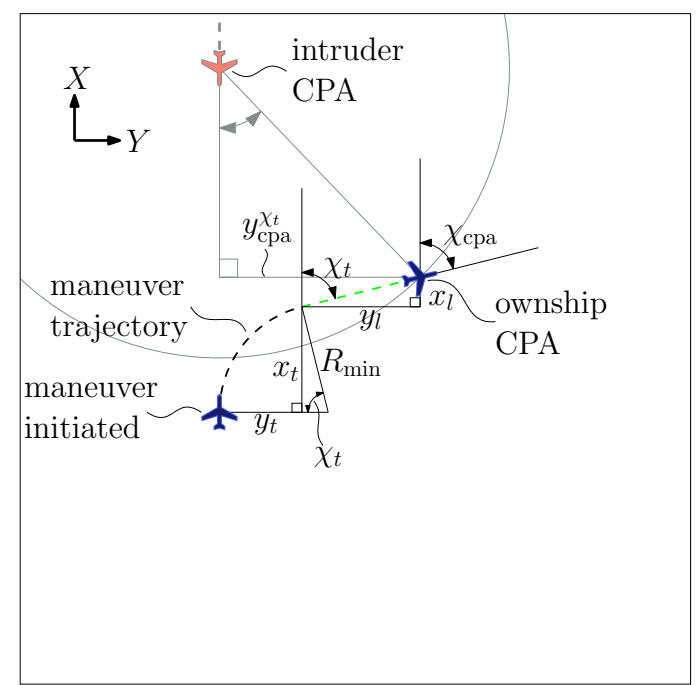

(a) Case 1: $y_{t} \leq y_{\mathrm{cpa}}^{\chi_{t}}$; ownship completes turning maneuver before reaching the CPA location.

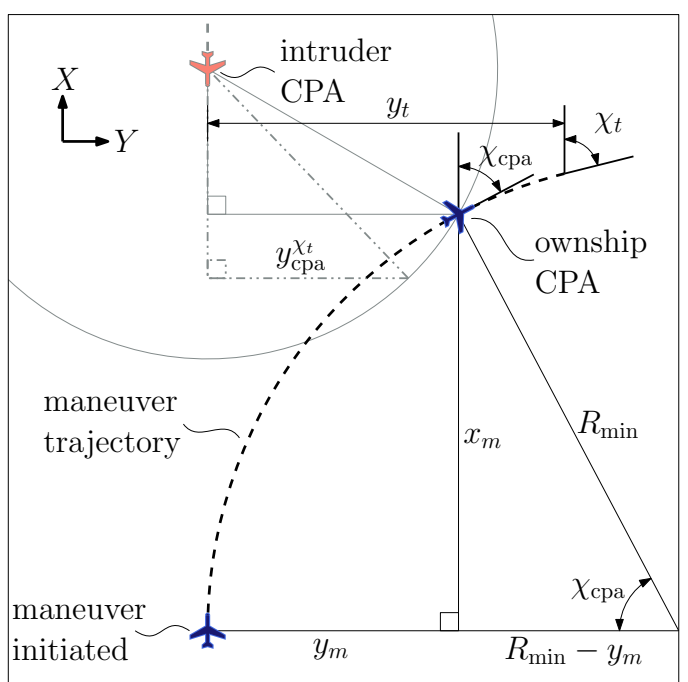

(b) Case 2: $y_{t}>y_{\mathrm{cpa}}^{\chi_{t}}$; ownship reaches CPA location before completing turning maneuver.

Fig. 6 Geometric diagram for the CPA location resulting from circular turning trajectories.

These cases can be distinguished mathematically in the following manner. First, we assume the ownship executes a turning maneuver until its course is equal to the prescribed value for $\chi_{t}$. The $Y$ component of this turning maneuver for both cases is calculated from $y_{t}$ as

$$
y_{t}=R_{\min }\left(1-\cos \chi_{t}\right)
$$

Second, we calculate $y_{\text {cpa }}$ with the course set equal to the desired turn angle $\chi_{t}$ and define this as $y_{\mathrm{cpa}}^{\chi_{t}}$. The exact derivation of this parameter will be shown in the subsection for case 1 (Sec. II B 3case 1). This $Y$ coordinate denotes the transition between case 1 and case 2 where the turn maneuver is completed at the exact moment the CPA is reached. The final step is to compare the calculated $y_{t}$ and $y_{\text {cpa }}^{\chi_{t}}$, which allows us to determine which geometry should be used to calculate the minimum detection range. For case $1, y_{t} \leq y_{\mathrm{cpa}}^{\chi_{t}}$, whereas for case $2, y_{t}>y_{\mathrm{cpa}}^{\chi_{t}}$.

As stated previously, for the GVV method, Eq. (7) can be expressed in terms of the variable $\theta_{\text {cpa }}$. Two of the variables $x_{\text {cpa }}$ and $y_{\text {cpa }}$ can be immediately expressed in terms of $\theta_{\text {cpa }}$ as

$$
\begin{aligned}
& x_{\mathrm{cpa}}=R_{s} \cos \theta_{\mathrm{cpa}}, \\
& y_{\mathrm{cpa}}=R_{s} \sin \theta_{\mathrm{cpa}} .
\end{aligned}
$$


Substituting these into Eq. (7) and simplifying results in

$$
0=v_{o} \sin \chi_{\mathrm{cpa}} \sin \theta_{\mathrm{cpa}}-\left(v_{i}+v_{o} \cos \chi_{\mathrm{cpa}}\right) \cos \theta_{\mathrm{cpa}} .
$$

The variable $\chi_{\mathrm{cpa}}$ is different for cases 1 and 2 and will be derived in the following two subsections (Secs. II B 3-case 1 and II B 3-case 2), along with the resulting minimum detection range:

\section{Case 1: $y_{t} \leq y_{\text {cpa }}^{\chi_{t}}$}

In case 1 , the ownship completes its turning maneuver before reaching the CPA; therefore, the course of the ownship when it reaches the CPA, $\chi_{\text {cpa }}$, will be equal to the prescribed value $\chi_{t}$. Substituting this into Eq. (27) results in

$$
0=v_{o} \sin \chi_{t} \sin \theta_{\text {cpa }}-\left(v_{i}+v_{o} \cos \chi_{t}\right) \cos \theta_{\text {cpa }} .
$$

This equation is then used to solve for $\theta_{\text {cpa }}$ as

$$
\theta_{\text {cpa }}=\tan ^{-1}\left(\frac{v_{i}+v_{o} \cos \chi_{t}}{v_{o} \sin \chi_{t}}\right),
$$

where $\theta_{\text {cpa }}$ is now used in the expressions for $x_{\text {cpa }}$, and $y_{\text {cpa }}$ to produce

$$
\begin{aligned}
& x_{\mathrm{cpa}}=R_{s} \frac{v_{o} \sin \chi_{t}}{\sqrt{v_{o}^{2}+v_{i}^{2}+2 v_{o} v_{i} \cos \chi_{t}}}, \\
& y_{\mathrm{cpa}}=R_{s} \frac{v_{i}+v_{o} \cos \chi_{t}}{\sqrt{v_{o}^{2}+v_{i}^{2}+2 v_{o} v_{i} \cos \chi_{t}}} .
\end{aligned}
$$

Because the ownship reaches a course of $\chi_{t}$ before reaching the CPA in case 1 , then the variable $y_{\text {cpa }}^{\chi_{t}}$ is equivalent to $y_{\text {cpa }}$ defined by Eq. (30):

$$
y_{\mathrm{cpa}}^{\chi_{t}}=R_{s} \frac{v_{i}+v_{o} \cos \chi_{t}}{\sqrt{v_{o}^{2}+v_{i}^{2}+2 v_{o} v_{i} \cos \chi_{t}}} .
$$

This value of $y_{\text {cpa }}^{\chi_{t}}$ is used to determine whether a specific encounter scenario is of case 1 or case 2 geometry.

Next, we define the variables $x_{t}, y_{t}, x_{l}$, and $y_{l}$ from the geometry of Fig. 6(a) as

$$
\begin{aligned}
& x_{t}=R_{\min } \sin \chi_{t}, \\
& y_{t}=R_{\min }\left(1-\cos \chi_{t}\right), \\
& y_{l}=y_{\mathrm{cpa}}-y_{t}=R_{s} \frac{v_{i}+v_{o} \cos \chi_{t}}{\sqrt{v_{o}^{2}+v_{i}^{2}+2 v_{o} v_{i} \cos \chi_{t}}}-R_{\min }\left(1-\cos \chi_{t}\right), \\
& x_{l}=y_{l} \cot \chi_{t}=\left[R_{s} \frac{v_{i}+v_{o} \cos \chi_{t}}{\sqrt{v_{o}^{2}+v_{i}^{2}+2 v_{o} v_{i} \cos \chi_{t}}}-R_{\min }\left(1-\cos \chi_{t}\right)\right] \cot \chi_{t},
\end{aligned}
$$


Using these definitions, we can define the remaining variables needed for the minimum detection range $x_{m}$ and $t_{m}$ as

$$
\begin{aligned}
x_{m} & =x_{t}+x_{l}, \\
& =R_{\min } \sin \chi_{t}+\left[R_{s} \frac{v_{i}+v_{o} \cos \chi_{t}}{\sqrt{v_{o}^{2}+v_{i}^{2}+2 v_{o} v_{i} \cos \chi_{t}}}-R_{\min }\left(1-\cos \chi_{t}\right)\right] \cot \chi_{t}, \\
t_{m}=\frac{L}{v_{o}}= & \frac{R_{\min \chi_{t}}+\sqrt{x_{l}^{2}+y_{l}^{2}}}{v_{o}} \\
= & \frac{R_{\min } \chi_{t}+\left[R_{s} \frac{v_{i}+v_{o} \cos \chi_{t}}{\sqrt{v_{o}^{2}+v_{i}^{2}+2 v_{o} v_{i} \cos \chi_{t}}}-R_{\min }\left(1-\cos \chi_{t}\right)\right] \sqrt{1+\cot ^{2} \chi_{t}}}{v_{o}},
\end{aligned}
$$

where $L$ is the length of the avoidance path of the ownship during the turning and straight segments of the maneuver.

Substituting Eqs. (29), (36), and (35) into Eq. (8), and using the relationship defined in Eq. (2) for $R_{\min }$, produces the final minimum detection range equation for case 1 as

$$
\begin{aligned}
& d_{\mathrm{MDR}}=\left(v_{o}+v_{i}\right) t_{c}+R_{\min } \sin \chi_{t}+\left[R_{s} \frac{v_{i}+v_{o} \cos \chi_{t}}{\sqrt{v_{o}^{2}+v_{i}^{2}+2 v_{o} v_{i} \cos \chi_{t}}}-R_{\min }\left(1-\cos \chi_{t}\right)\right] \cot \chi_{t} \\
& +\frac{v_{i}}{v_{o}}\left[R_{\min } \chi_{t}+\left[R_{s} \frac{v_{i}+v_{o} \cos \chi_{t}}{\sqrt{v_{o}^{2}+v_{i}^{2}+2 v_{o} v_{i} \cos \chi_{t}}}-R_{\min }\left(1-\cos \chi_{t}\right)\right] \sqrt{1+\cot ^{2} \chi_{t}}\right] \\
& +R_{s} \frac{v_{o} \sin \chi_{t}}{\sqrt{v_{o}^{2}+v_{i}^{2}+2 v_{o} v_{i} \cos \chi_{t}}} \text {. }
\end{aligned}
$$

Case 2: $y_{t}>y_{\text {cpa }}^{\chi_{t}}$

For this case, we begin with Eq. (27); however, we must define expressions for $\cos \chi_{\text {cpa }}$ and $\sin \chi_{\text {cpa }}$ as functions of $\theta_{\text {cpa }}$. We first define expressions for $y_{m}$ and $x_{m}$ as

$$
\begin{aligned}
& y_{m}=y_{\mathrm{cpa}}=R_{s} \sin \theta_{\mathrm{cpa}}, \\
& x_{m}=\sqrt{R_{\mathrm{min}}^{2}-\left(R_{\mathrm{min}}-y_{m}\right)^{2}}=\sqrt{R_{s} \sin \theta_{\mathrm{cpa}}\left(2 R_{\mathrm{min}}-R_{s} \sin \theta_{\mathrm{cpa}}\right)} .
\end{aligned}
$$

These values for $y_{m}$ and $x_{m}$ can now be used to define $\cos \chi_{\text {cpa }}$ and $\sin \chi_{\text {cpa }}$ as

$$
\begin{aligned}
& \cos \left(\chi_{\mathrm{cpa}}\right)=\frac{R_{\mathrm{min}}-y_{m}}{R_{\mathrm{min}}}=\frac{R_{\mathrm{min}}-R_{s} \sin \theta_{\mathrm{cpa}}}{R_{\mathrm{min}}}, \\
& \sin \left(\chi_{\mathrm{cpa}}\right)=\frac{x_{m}}{R_{\mathrm{min}}}=\frac{\sqrt{R_{s} \sin \theta_{\mathrm{cpa}}\left(2 R_{\mathrm{min}}-R_{s} \sin \theta_{\mathrm{cpa}}\right)}}{R_{\min }} .
\end{aligned}
$$


Similarly, an expression for $\chi_{\mathrm{cpa}}$ can be defined as

$$
\chi_{\mathrm{cpa}}=\tan ^{-1}\left(\frac{x_{m}}{R_{\mathrm{min}}-y_{m}}\right)=\tan ^{-1}\left(\frac{\sqrt{R_{s} \sin \theta_{\mathrm{cpa}}\left(2 R_{\mathrm{min}}-R_{s} \sin \theta_{\mathrm{cpa}}\right)}}{R_{\mathrm{min}}-R_{s} \sin \theta_{\mathrm{cpa}}}\right) .
$$

Substituting these expressions from Eqs. (40) and (41) into Eq. (27) and manipulating produces an equation in terms of a single parameter $\theta_{\text {cpa }}$ in the following form:

$$
0=a \sin ^{3} \theta_{\mathrm{cpa}}+b \sin ^{2} \theta_{\mathrm{cpa}}+c \sin \theta_{\mathrm{cpa}}+d,
$$

where $a, b, c$, and $d$ are defined as

$$
\begin{aligned}
& a=2 v_{i} v_{o} R_{\min } R_{s}, \\
& b=v_{o}^{2} R_{s}^{2}-\left(v_{i}+v_{o}\right)^{2} R_{\min }^{2}, \\
& c=-2 v_{o}\left(v_{i}+v_{o}\right) R_{\min } R_{s}, \\
& d=\left(v_{i}+v_{o}\right)^{2} R_{\min }^{2} .
\end{aligned}
$$

Equation (43) has been formulated to be cubic in $\sin \theta_{\text {cpa }}$. Applying Cardano's formulas [16] and expressing $\sin \theta_{\text {cpa }}$ as $z$ produces three roots for $\sin \theta_{\text {cpa }}$ as

$$
\begin{aligned}
& z_{1}=-\frac{b}{3 a}+(S+T), \\
& z_{2}=-\frac{b}{3 a}-\frac{1}{2}(S+T)+\frac{1}{2} i \sqrt{3}(S-T), \\
& z_{3}=-\frac{b}{3 a}-\frac{1}{2}(S+T)-\frac{1}{2} i \sqrt{3}(S-T),
\end{aligned}
$$

where $S$ and $T$ are defined as $S=\sqrt[3]{R+\sqrt{D}}$ and $T=\sqrt[3]{R-\sqrt{D}}$, respectively; $D$ is defined as $D=Q^{3}+R^{2}$; and $Q$ and $R$ are defined as

$$
Q=\frac{c}{3 a}-\left(\frac{b}{3 a}\right)^{2}
$$

and

$$
R=\frac{b c}{6 a^{2}}-\frac{d}{2 a}-\left(\frac{b}{3 a}\right)^{3}
$$

respectively. Because we are trying to find a root for the expression $\sin \theta_{\text {cpa }}$, the root must first lie within the bounds $[-1,1]$. Second, the solution for $\theta_{\text {cpa }}$ must lie within the bounds of $[0,90]$ deg due 
to the head-on approach of the ownship and intruder and the right turning maneuver of the ownship as seen in Fig. 6, which means the root must further be restricted to $[0,1]$. Finally, there may still exist multiple roots within the bounds $[0,1]$; therefore, a third constraint must be satisfied. The chosen root must produce a value of $\theta_{\text {cpa }}$ that, when used to calculate $\chi_{\text {cpa }}$ in Eq. (42), produces a value within the bounds of $[0,90]$ deg. Once these constraints are satisfied, the true root is identified and used to find $\theta_{\text {cpa }}$ as

$$
\theta_{\text {cpa }}=\sin ^{-1} z
$$

This value of $\theta_{\text {cpa }}$ is now used to define values for $x_{\text {cpa }}$ and $y_{\text {cpa }}$, shown in Eqs. (25) and (26), and $y_{m}$ and $x_{m}$, shown in Eqs. (38) and (39). The time to maneuver is found as

$$
t_{m}=\frac{L}{v_{o}}=\frac{R_{\mathrm{min}} \chi_{\mathrm{cpa}}}{v_{o}}=\frac{1}{v_{o}} R_{\mathrm{min}} \tan ^{-1}\left(\frac{\sqrt{R_{s} \sin \theta_{\mathrm{cpa}}\left(2 R_{\mathrm{min}}-R_{s} \sin \theta_{\mathrm{cpa}}\right)}}{R_{\mathrm{min}}-R_{s} \sin \theta_{\mathrm{cpa}}}\right) .
$$

Substituting Eqs. (25), (39), and (45) into Eq. (8) produces the final equation for the case 2 minimum detection range as

$$
\begin{aligned}
d_{\mathrm{MDR}} & =\left(v_{o}+v_{i}\right) t_{c}+\sqrt{R_{s} \sin \theta_{\mathrm{cpa}}\left(2 R_{\mathrm{min}}-R_{s} \sin \theta_{\mathrm{cpa}}\right)} \\
& +\frac{v_{i}}{v_{o}} R_{\mathrm{min}} \tan ^{-1}\left(\frac{\sqrt{R_{s} \sin \theta_{\mathrm{cpa}}\left(2 R_{\mathrm{min}}-R_{s} \sin \theta_{\mathrm{cpa}}\right)}}{R_{\mathrm{min}}-R_{s} \sin \theta_{\mathrm{cpa}}}\right)+R_{s} \cos \theta_{\mathrm{cpa}}
\end{aligned}
$$

where $\theta_{\text {cpa }}$ is defined in Eq. (44), and $R_{\text {min }}$ is defined in Eq. (2).

\section{Results: Method Comparison and Validation}

With the equations for the minimum detection range derived, we now present results showing calculated values for the minimum detection range as a function of each of the parameters used in the equations. We present these results for the two methods developed in this paper: the TGVV and GVV methods. We also present results for the two prior methods, TT and GT, and provide a detailed comparison of all four methods. The parameters that are used by all four methods include $v_{o}, v_{i}, R_{s}, \phi_{\max }$, and $t_{c}$. For the TGVV and GVV methods, we have the additional parameter $\chi_{t}$; for the TGVV method, we have two parameters used to describe the bank-angle dynamics $\dot{\phi}_{\max }$ and $\tau$. In creating the results, a nominal set of values is chosen for each of these parameters, except the ownship speed, and are listed in Table 3 . 


\begin{tabular}{lccccccc}
\hline \hline Parameter & $v_{i}$ & $R_{s}$ & $\phi_{\max }$ & $t_{c}$ & $\chi_{t}$ & $\dot{\phi}_{\max }$ & $\tau$ \\
\hline Value & $150 \mathrm{kt}$ & $500 \mathrm{ft}$ & $30 \mathrm{deg}$ & $5.0 \mathrm{~s}$ & $90 \mathrm{deg}$ & $30 \mathrm{deg} / \mathrm{s}$ & $0.5 \mathrm{~s}$ \\
\hline \hline
\end{tabular}

Table 3 Nominal parameter values used in the calculation of $d_{\mathrm{MDR}}$.

The minimum detection range is calculated for all four methods using the parameter values from Table 3 and is shown in Fig. 7 . In this figure, $d_{\mathrm{MDR}}$ is plotted versus the ownship speed, with level curves used to vary one or two additional parameters.

In Fig. $7(\mathrm{a})$, we first compare various values for $\dot{\phi}_{\max }$ and $\tau$, which only affect the calculation of the TGVV method. The GVV method is also plotted to demonstrate what values of $\dot{\phi}_{\max }$ and $\tau$ result in the two methods producing similar values.

For Figs. $7(\mathrm{~b})-7(\mathrm{f})$, a single pair of values is used for $\dot{\phi}_{\max }$ and $\tau$, which results in a single set of level curves for the TGVV method. Upon careful inspection, it can be seen that each subplot contains one level that corresponds to the core set of parameters in Table 3, which results in one common level among each of the subplots. This allows us to see how the minimum detection range deviates from a common level as each parameter is changed. Figure $7(\mathrm{~b})$ is used to vary $v_{i}$, Fig. $7(\mathrm{c})$ varies $R_{s}$, Fig. $7(\mathrm{~d})$ varies $\phi_{\max }$, Fig. $7(\mathrm{e})$ varies $t_{c}$, and Fig. $7(\mathrm{f})$ varies $\chi_{t}$.

In Fig. 7, the right-pointing orange triangles are used to identify transition points from case B to case A for the TGVV method when viewed from left to right. Similarly, left-pointing black triangles are used to identify transition points from case 1 to case 2 for the GVV method when viewed from left to right.

From Fig. 7(a), a general trend between the TGVV and GVV methods can be seen. We see that, as $\dot{\phi}_{\max }$ increases and $\tau$ decreases, the TGVV method $d_{\mathrm{MDR}}$ values approach the same values as those from the GVV method, shown by the solid black line. It can be seen that the TGVV method always predicts a minimum detection range slightly larger than the GVV method, which results from including the bank-angle dynamics in the turning maneuver.

It is evident from Figs. 7(b)-7(f) that the TT and GT methods approach the GVV method for ownship speeds in the case 2 region of the GVV method. This is because case 2 of the GVV method uses an avoidance maneuver that is performed solely by turning, which is an assumption made in 
both the TT and GT methods. For ownship speeds in the case 1 region of the GVV method, the

TT and GT methods predict values for the minimum detection range significantly smaller than the

GVV and TGVV methods.
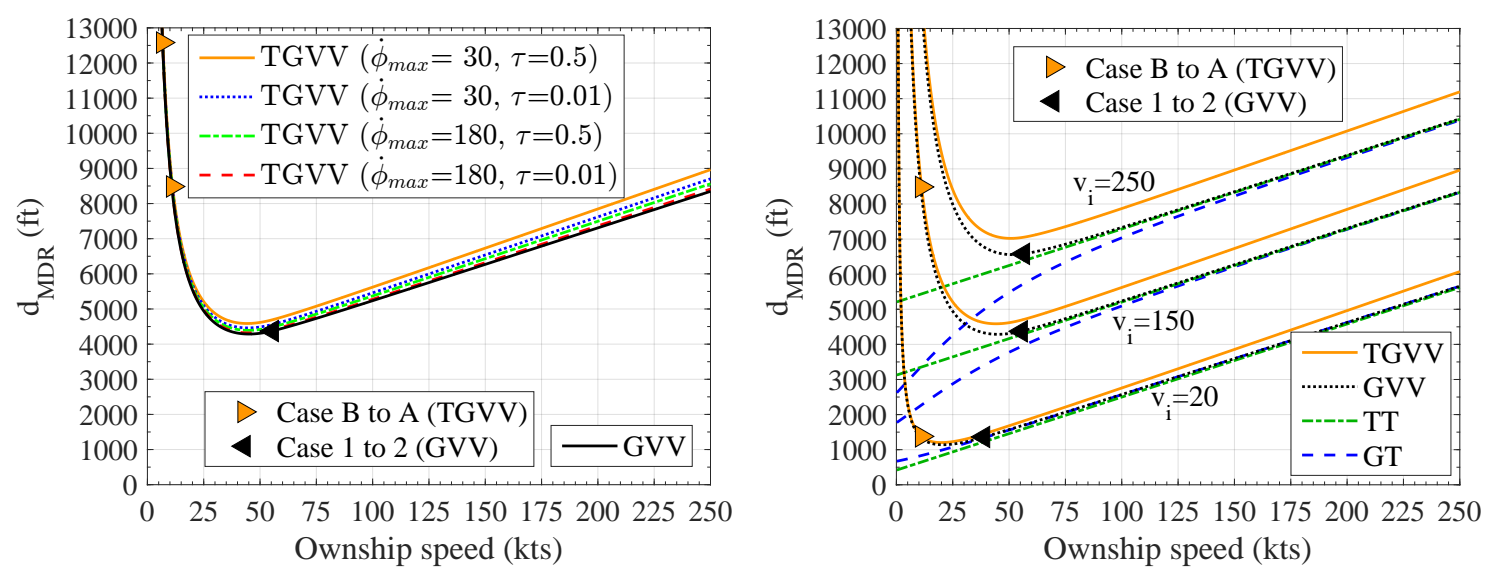

(a) Comparison as a function of $\dot{\phi}_{\max }$ and $\tau$.

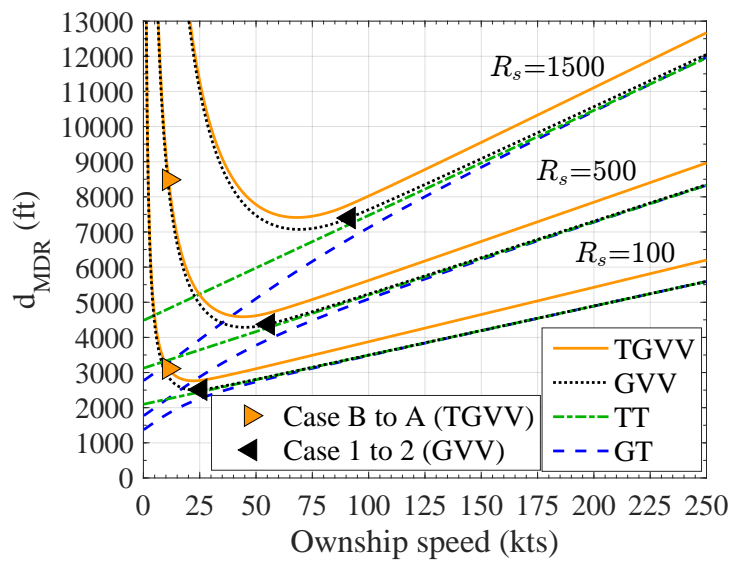

(b) Comparison as a function of $v_{i}$.

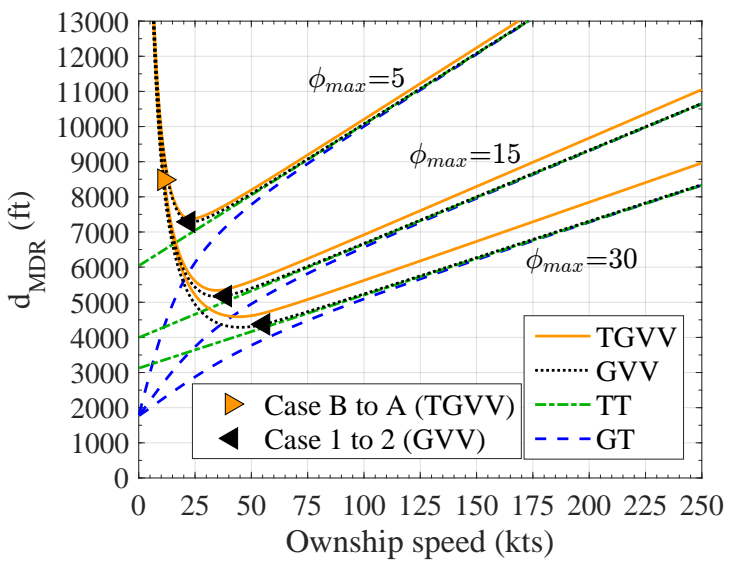

(c) Comparison as a function of $R_{s}$.

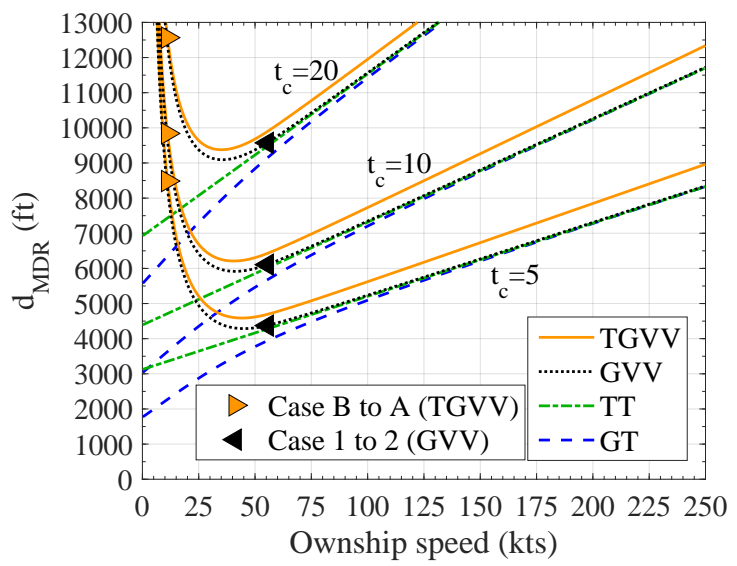

(d) Comparison as a function of $\phi_{\max }$.

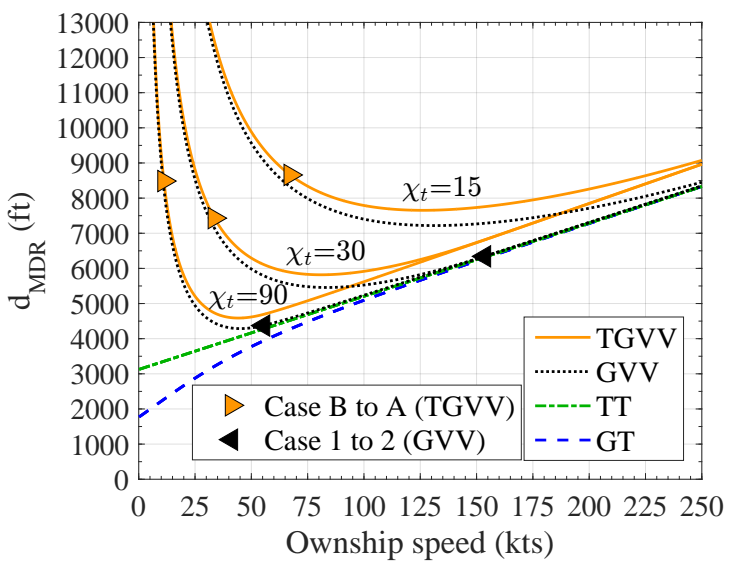

(e) Comparison as a function of $t_{c}$.

(f) Comparison as a function of $\chi_{t}$.

Fig. 7 Comparison of $d_{\mathrm{MDR}}$ as a function of $v_{o}, v_{i}, R_{s}, \phi_{\max }, t_{c}, \chi_{t}, \dot{\phi}_{\max }$ and $\tau$. 
Finally, from each subplot of Fig. 7, it can be seen that, as the ownship speed increases, each of the methods models $d_{\mathrm{MDR}}$ as a linear function of the ownship speed. For large values of $v_{o}$, the nonlinear geometric methods (GVV and GT) converge to the linear model predicted by the TT method. Using the equation for the minimum detection range from the TT method, the slope of these lines can be inferred from of Eq. 4 to be a function of $R_{s}, \phi_{\max }$, and $t_{c}$. As $R_{s}$ and $t_{c}$ increase, the slopes of the resulting lines increase, as can be seen in Figs. 7(c) and 7(e); however, as $\phi_{\max }$ increases, the slope of the resulting lines decrease, as can be seen in Fig. 7(d). As the remaining variables, $v_{i}$ and $\chi_{t}$, are increased, the slopes resulting from the geometric methods stay constant as shown in Figs. $7(\mathrm{~b})$ and $7(\mathrm{f})$.

Having calculated the minimum detection range, we must now determine the accuracy of the results. If the true minimum detection range is used to initialize the distance between two aircraft, the resulting CPA between the two aircraft will be exactly equal to the safety radius, $R_{s}$. This means we can check the accuracy of the minimum detection range calculated from each of the four methods by comparing the resulting CPA to the safety radius. To find the resulting CPA for each method, a simulation is performed. The ownship and intruder are initialized in a head-on configuration at a distance equal to $d_{\mathrm{MDR}}$. Both aircraft fly toward each other without maneuvering during the computation time; after which, the ownship begins turning using the bank-angle dynamics described by the TGVV method with the parameters $\dot{\phi}_{\max }, \tau, \phi_{\max }$, and $\chi_{t}$. Once the ownship has turned to the predefined $\chi_{t}$, it flies straight until it is far from the intruder. During the simulation, the relative range and CPA between the ownship and the intruder are calculated.

An example of the relative range is shown in Fig. 8. The parameters that are used come from Table 3 , and the value of the ownship speed is $v_{o}=25 \mathrm{kt}$. The TGVV method is shown by the solid orange line; at time zero, the relative range between the two aircraft is approximately $5209 \mathrm{ft}$. As the two aircraft continue their flight paths, the closest point of approach is exactly equal to $500 \mathrm{ft}$ at approximately $18.9 \mathrm{~s}$. Because the TGVV method uses the same dynamic model as the ownship in the CPA simulation, the predicted relative range from the TGVV method is identical to the simulation truth model. The GVV method is shown by the dotted black line, and the relative range predicted at time zero is approximately $4942 \mathrm{ft}$. In this case, the two aircraft reach a CPA 
of $456 \mathrm{ft}$ at approximately $17.9 \mathrm{~s}$, which is a violation of the safety volume. Similarly, the ownship flight paths from the TT and GT methods result in a penetration of the safety volume with CPAs of 243 and $116 \mathrm{ft}$ at approximately 12.9 and $9.9 \mathrm{~s}$, respectively.

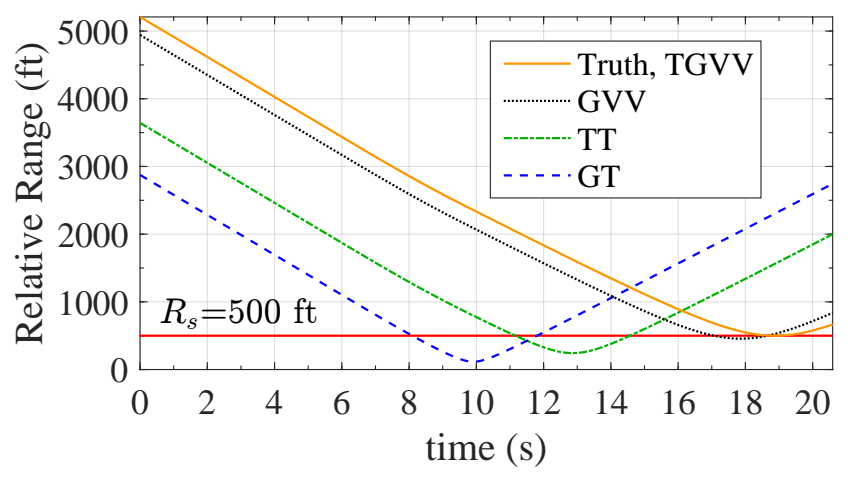

Fig. 8 Relative range vs. time and the resulting CPA.

The CPA is used to compare the accuracy of each of the four methods. In Fig. 9, the CPA is plotted versus the speed of the ownship. In Fig. 9(a), four different sets of parameters are used for the bank-angle dynamics of the ownship; whereas in the other subplots, only the nominal set of parameters is used for the bank-angle dynamics of the ownship and other critical parameters are varied. The first observation to make from each of the subplots is that the TGVV method always produces a CPA exactly equal to the value chosen for $R_{s}$, which means that the safety volume has not been penetrated and the true minimum detection range has been found. Additionally, the GVV, TT, and GT methods all produce a CPA less than the chosen value for $R_{s}$, which means the safety volume has been penetrated and the calculated minimum detection range is an underapproximation. Fig. 9(a), however, shows that the CPA of the GVV method approaches the safety radius of $500 \mathrm{ft}$ as $\dot{\phi}_{\max }$ increases; and $\tau$ decreases, implying that the GVV method becomes a good approximation of the TGVV method as the speed of the bank-angle response increases.

The next critical observation to make is seen in Fig. 9(b), which shows that the CPA of the TT and GT methods converge to the CPA of the GVV method as $v_{o}$ increases. Additionally, it can be seen that the GT method approaches the GVV method as $v_{i}$ decreases. This is because the underlying assumption of tangent turning circles is more reasonable when the intruder speed is small relative to the ownship speed. 


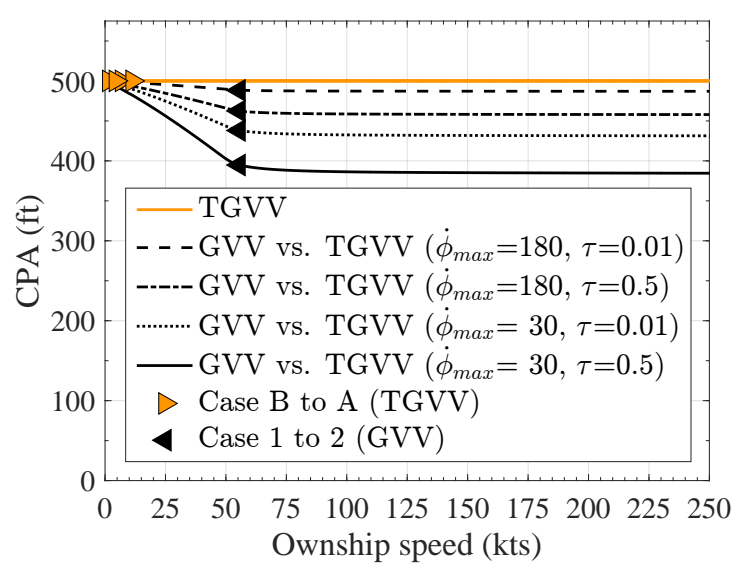

(a) Comparison as a function of $\dot{\phi}_{\max }$ and $\tau$.

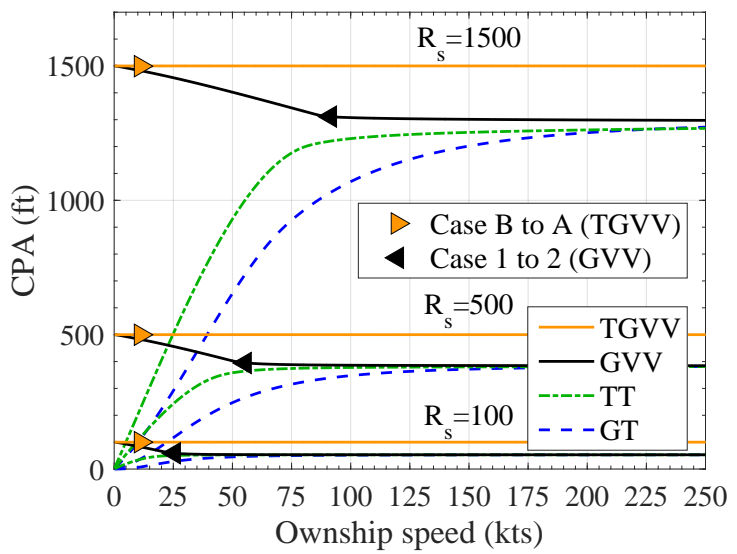

(c) Comparison as a function of $R_{s}$.

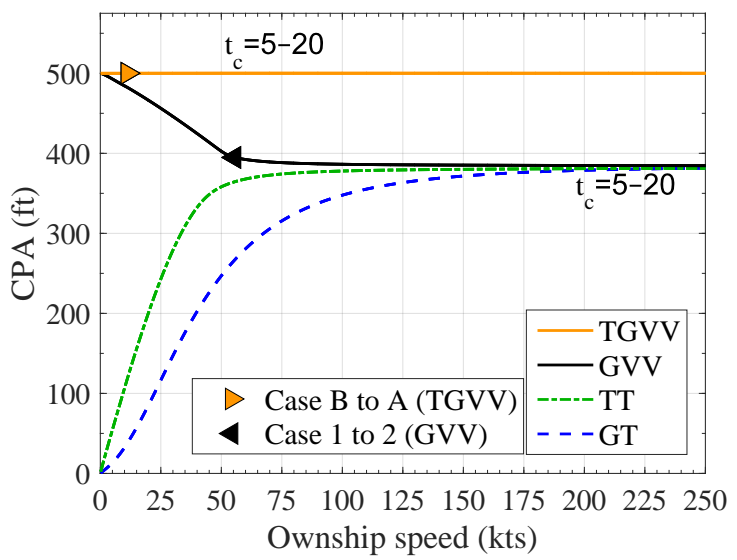

(e) Comparison as a function of $t_{c}$.

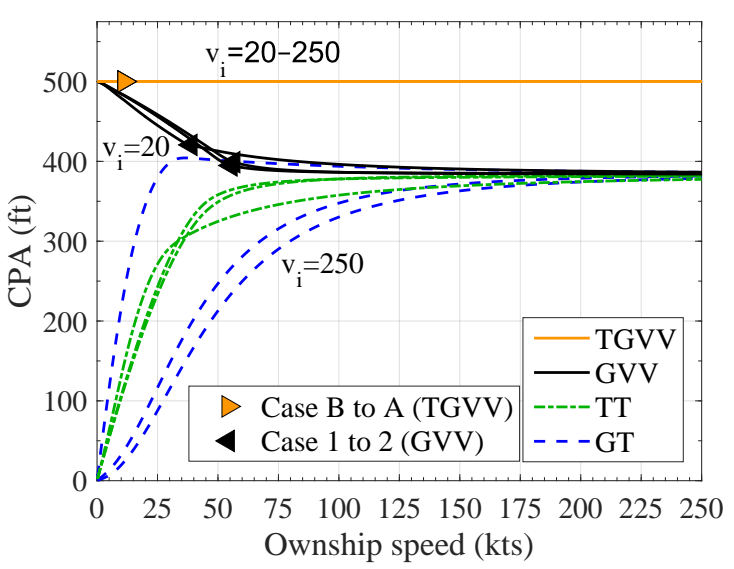

(b) Comparison as a function of $v_{i}$.

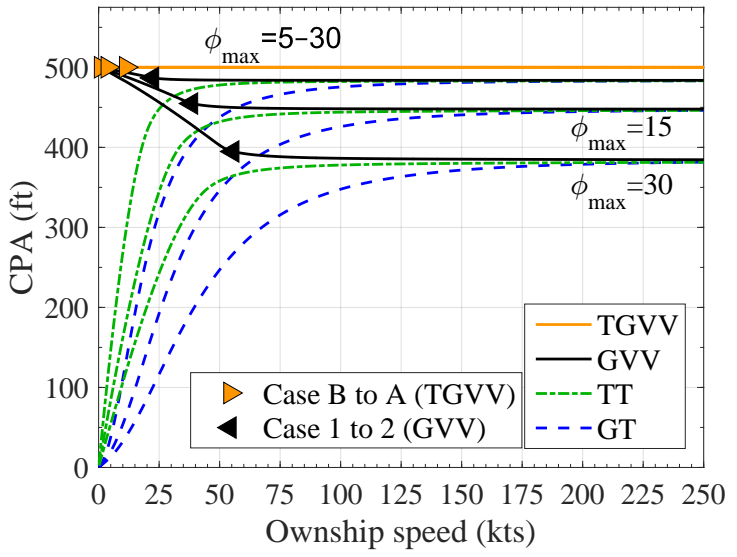

(d) Comparison as a function of $\phi_{\max }$.

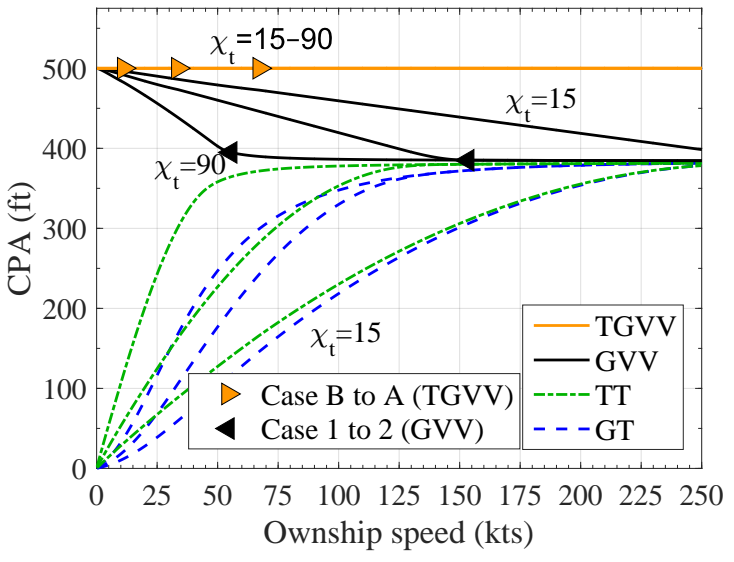

(f) Comparison as a function of $\chi_{t}$.

Fig. 9 Comparison of the CPA as a function of $v_{o}, v_{i}, R_{s}, \phi_{\max }, t_{c}, \chi_{t}, \dot{\phi}_{\max }$ and $\tau$.

Figure 9(d) shows that the CPA of the GVV, TT, and GT methods approaches the desired value chosen for $R_{s}$ as $\phi_{\max }$ decreases. This is because smaller values of $\phi_{\max }$ result in shorter bank-angle transients and produce more circular turns that more closely match the circular-turn assumptions 
of the GVV, TT, and GT methods.

Finally, from Fig. 9(f), the TT and GT methods depart from the GVV method as $\chi_{t}$ decreases at low ownship speeds. This is because the TT and GT methods do not provide any compensation for limiting the turn angle, $\chi_{t}$. Instead, these methods assume the ownship is always in a banked turning maneuver as it passes the intruder. This assumption matches the avoidance trajectory of the ownship for case 2 of the GVV method and is a valid assumption for large ownship speeds. This assumption causes issues at low ownship speeds because the ownship completes its turning maneuver before reaching the CPA, which corresponds to case 1 of the GVV method.

In this simulation the TGVV method results in a CPA matching the safety radius, which means the true minimum detection range has been found. The geometric methods (GVV, TT, and GT), however, are computationally simpler, and it is of interest to know how well they approximate the solution produced by the TGVV method. Figure 10 provides this information by showing the percent relative error in the minimum detection range between the other methods and the TGVV method.

From each subplot in Fig. 10, we see that the GVV method produces the smallest relative error. We also see that the TT method generally produces a relative error less than the GT method except when $v_{i}$ approaches zero, as seen in Fig. 10(b). In Fig. 10(a), the relative error in $d_{\mathrm{MDR}}$ is plotted versus $1 / \dot{\phi}_{\max }$ and $\tau$, with the remaining parameters fixed at their nominal values and with the ownship speed equal to $150 \mathrm{kt}$. The value of $\tau$ is varied between 0 and $1 \mathrm{~s}$, whereas $\dot{\phi}_{\max }$ is varied from $30 \mathrm{deg} / \mathrm{s}$ to infinity. Plotting against the inverse of $\dot{\phi}_{\max }$ improves the presentation of the data and facilitates interpretation of the result. By continuously varying $\dot{\phi}_{\max }$ and $\tau$, the effects of each variable on the accuracy of the GVV method can be more easily seen. From the subplot, it can be seen that the relative error does decrease as $\tau$ decreases; however, it does not decrease to zero. As the inverse of $\dot{\phi}_{\max }$ decreases, however, the relative error does go to zero for all values of $\tau$. This shows that, as the bank-angle maneuver of the ownship becomes more instantaneous, the results of the geometric GVV method converge to those of the TGVV method.

The results presented thus far show that the TT and GT methods converge to the GVV method for large ownship speeds relative to the intruder speed, and the GVV method converges to the TGVV 


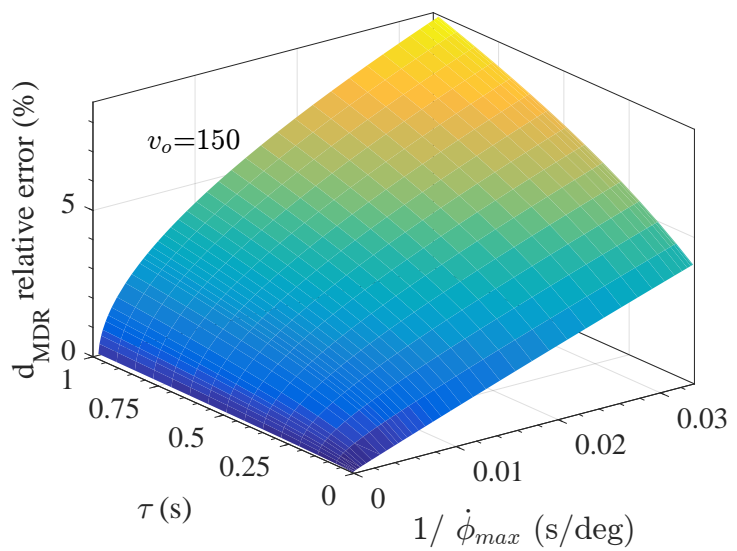

(a) GVV relative to TGVV as a function of $\dot{\phi}_{\max }$ and $\tau$.

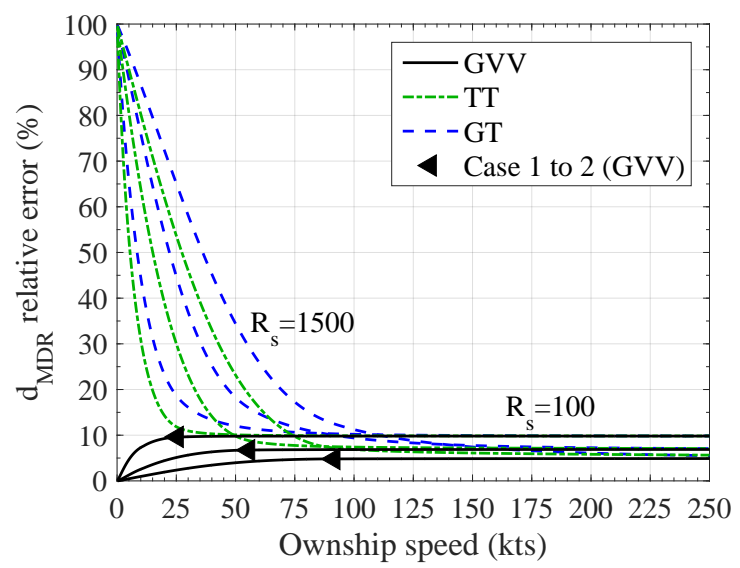

(c) Relative to TGVV as a function of $R_{s}$.

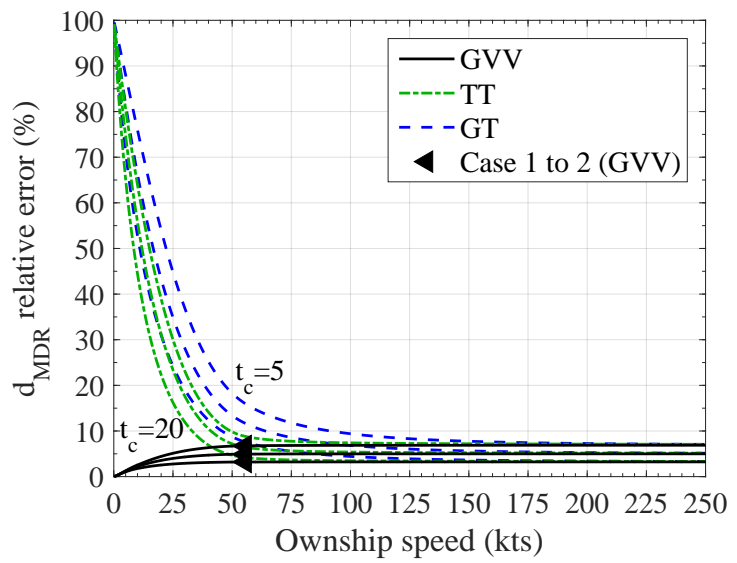

(e) Relative to TGVV as a function of $t_{c}$.

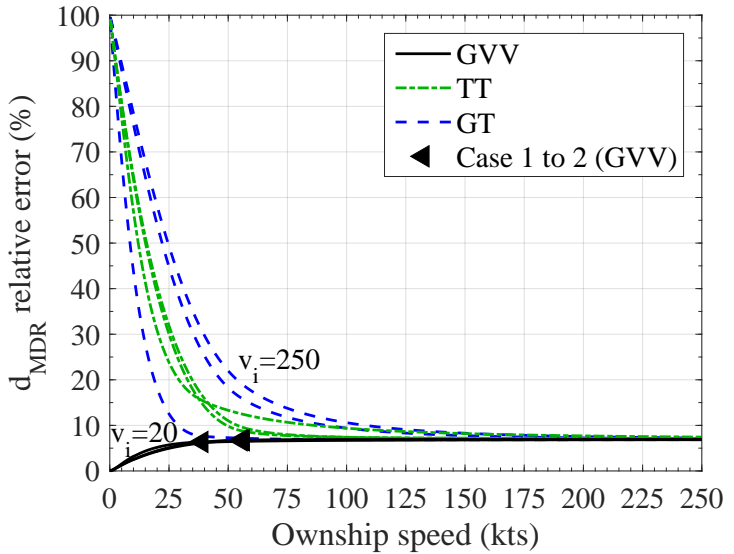

(b) Relative to TGVV as a function of $v_{i}$.

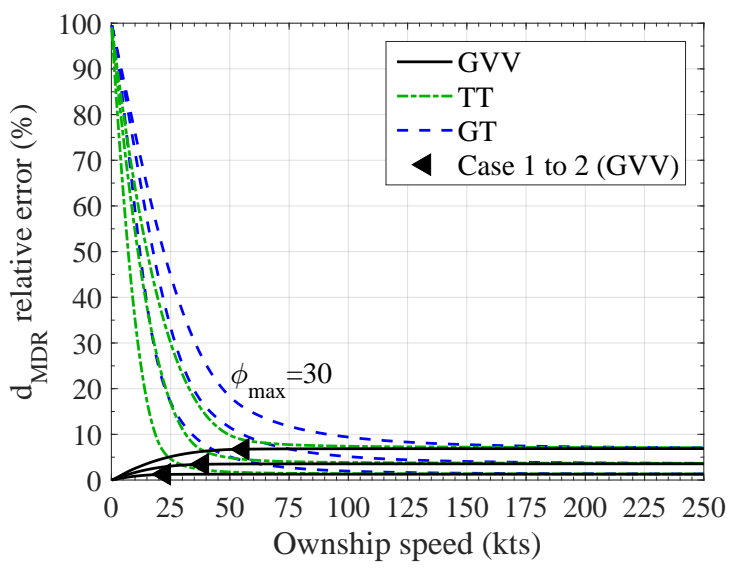

(d) Relative to TGVV as a function of $\phi_{\max }$.

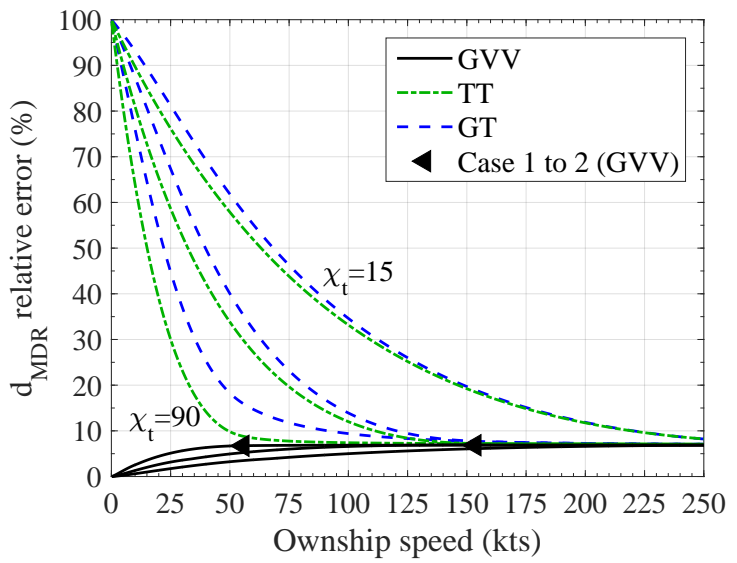

(f) Relative to TGVV as a function of $\chi_{t}$.

Fig. 10 Percent relative error of $d_{\mathrm{MDR}}$ as a function of $v_{o}, v_{i}, R_{s}, \phi_{\max }, t_{c}, \chi_{t}, \dot{\phi}_{\max }$ and $\tau$.

method for fast bank-angle transients and small bank angles. We can thus conclude that the TT and GT methods provide good approximations for the minimum detection range at large ownship speeds relative to the intruder speed, fast bank-angle transients, and small bank angles. 
Although the main focus of this paper is on methods for the calculation of minimum detection range, the time to maneuver is a quantity of significant importance and accompanying results for this parameter are presented in Fig. 11 .

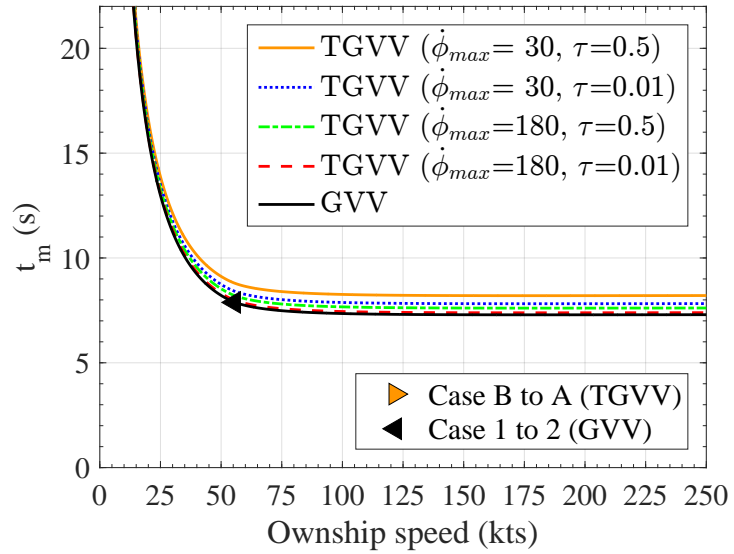

(a) Comparison as a function of $\dot{\phi}_{\max }$ and $\tau$.

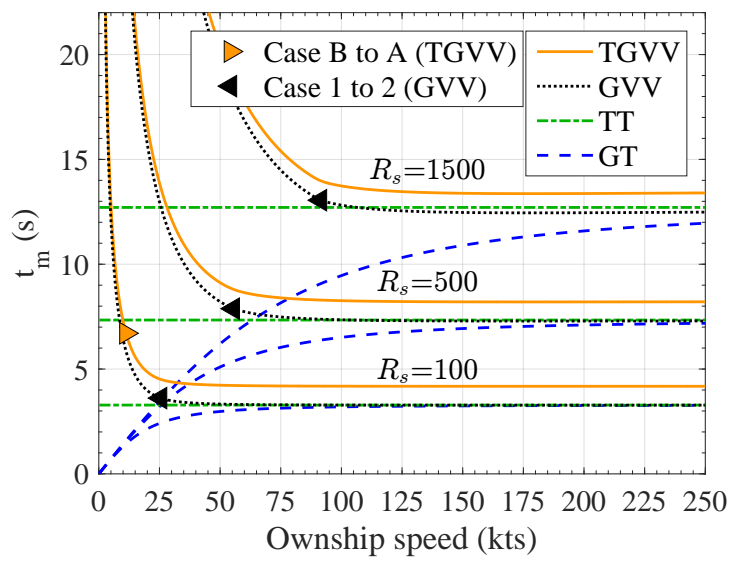

(c) Comparison as a function of $R_{s}$.

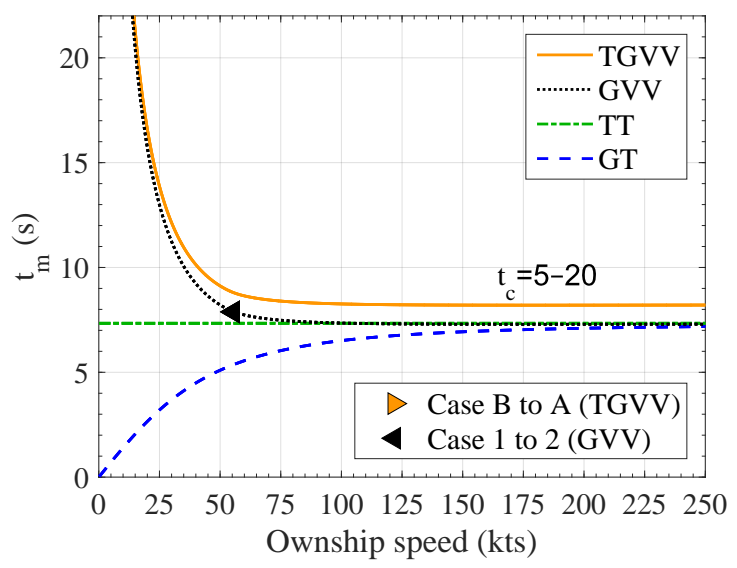

(e) Comparison as a function of $t_{c}$.

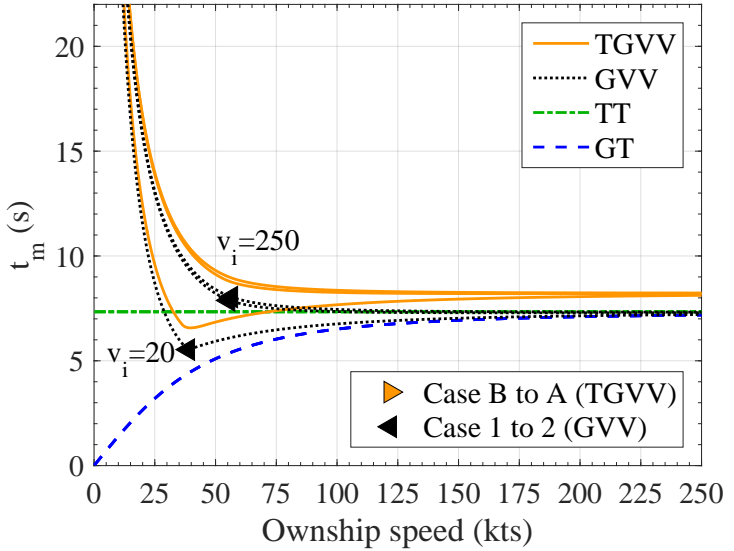

(b) Comparison as a function of $v_{i}$.

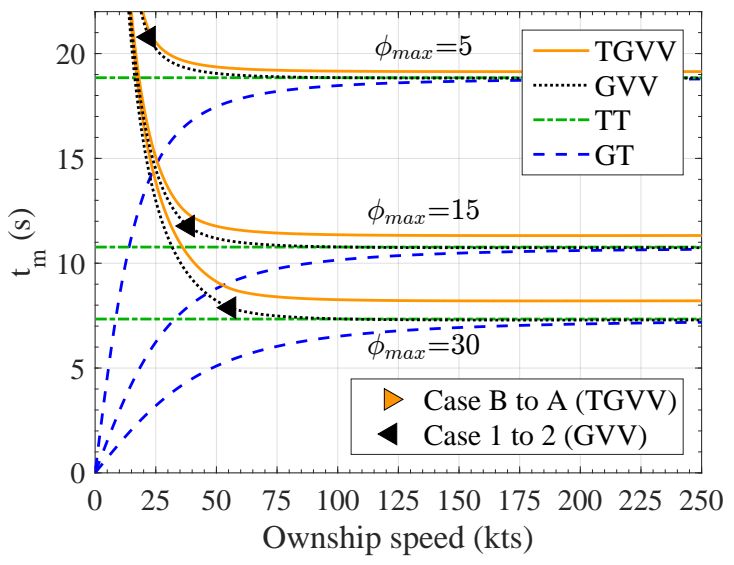

(d) Comparison as a function of $\phi_{\max }$.

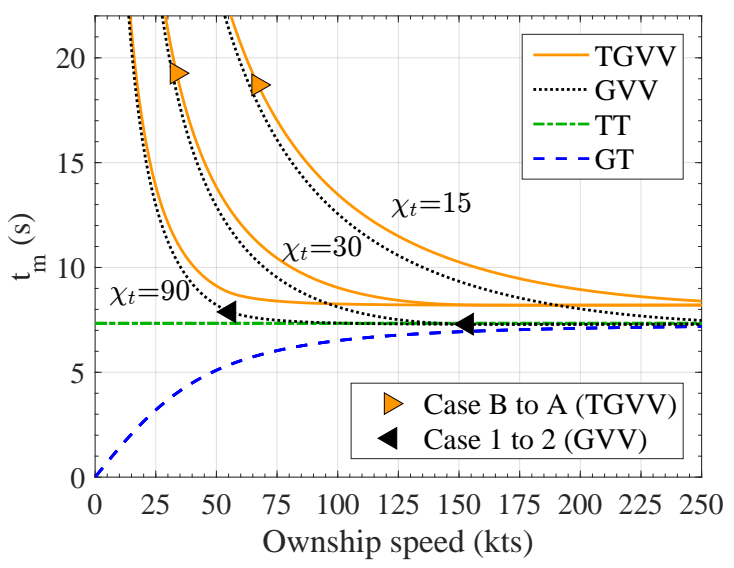

(f) Comparison as a function of $\chi_{t}$.

Fig. 11 Comparison of $t_{m}$ as a function of $v_{o}, v_{i}, R_{s}, \phi_{\max }, t_{c}, \chi_{t}, \dot{\phi}_{\max }$ and $\tau$. 
As stated previously, the minimum detection range of the geometric methods (GVV, TT, and GT) approach linear functions for large ownship speeds. From Fig. 11, a similar observation can be made for the time to maneuver. The TT method predicts a maneuver time that is constant with ownship speed. Comparing Eqs. 3 and 4 , and noting that the maneuver time $t_{m}$ is the same as the turn time $t_{t}$ for the TT method, shows that the predicted TT maneuver time is

$$
\sqrt{\frac{2 R_{s} \cot \phi_{\max }}{g}}
$$

which is independent of the ownship speed. For large ownship speeds, the GT and GVV methods converge to the maneuver time value predicted by the TT method. In Fig. 11(c), we see that, as $R_{s}$ increases, the predicted maneuver times also increase. From Fig. 11(d), we see that, as $\phi_{\max }$ increases, the maneuver times decrease.

From Figs. 11(b) and 11(f), we see that the TGVV and GVV methods account for variations $v_{i}$ and $\chi_{t}$, as seen by three sets of lines; whereas the TT and GT methods do not account for them. This agrees with the expressions for the predicted time to maneuver in the TT and GT methods where the predicted $t_{m}$ for the GT method can be derived from Eq. (5) as

$$
\frac{v_{o}}{g \tan \phi_{\max }} \cos ^{-1}\left(\frac{v_{o}^{2}}{v_{o}^{2}+R_{s} g \tan \phi_{\max }}\right) .
$$

The results presented thus far have used parameter values consistent with a collision-avoidance encounter. Results are now presented for parameter values more consistent with a self-separation scenario. These results use the following parameters: $R_{s}=0.75 \mathrm{n}$ mile, which is the lateral UAS well-clear requirement; $\phi_{\max }=5 \mathrm{deg} ; \chi_{t}=15 \mathrm{deg} ; t_{c}=20 \mathrm{~s}$, which includes tracking and typical pilot response delay with air traffic control interaction; $\dot{\phi}_{\max }=10 \mathrm{deg} / \mathrm{s} ; \tau=0.5 \mathrm{~s} ; v_{o}=0-1250 \mathrm{kt}$; and $v_{i}=250,500,750,1000$, and $1250 \mathrm{kt}$. The results for these parameters are shown in Fig. 12.

From Fig. 12(a), it can be seen that the the minimum detection range is now on the order of 10 to $50 \mathrm{n}$ miles instead of 2000 to 10,000 ft. Figure 12(b) shows that the CPA of the TGVV method is equal to the value chosen for $R_{s}$ of $0.75 \mathrm{n}$ mile. We also see that the GVV method produces a CPA close to the desired value for $R_{s}$. In Fig. 12(c), we see that the relative error of the GVV method stays well under $1 \%$. Finally, in Fig. 12(d), we see that, for large ownship speeds, the time to maneuver has increased to about $57 \mathrm{~s}$, as would be expected for the self-separation simulation 


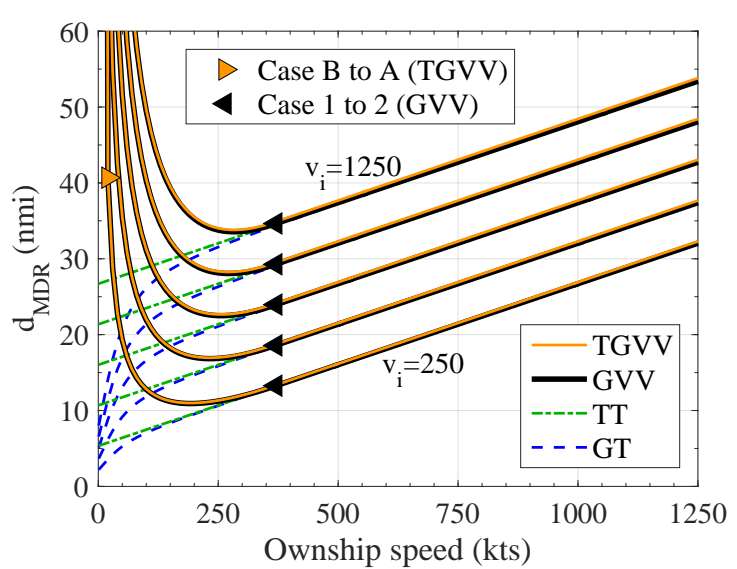

(a) Comparison of $d_{\mathrm{MDR}}$

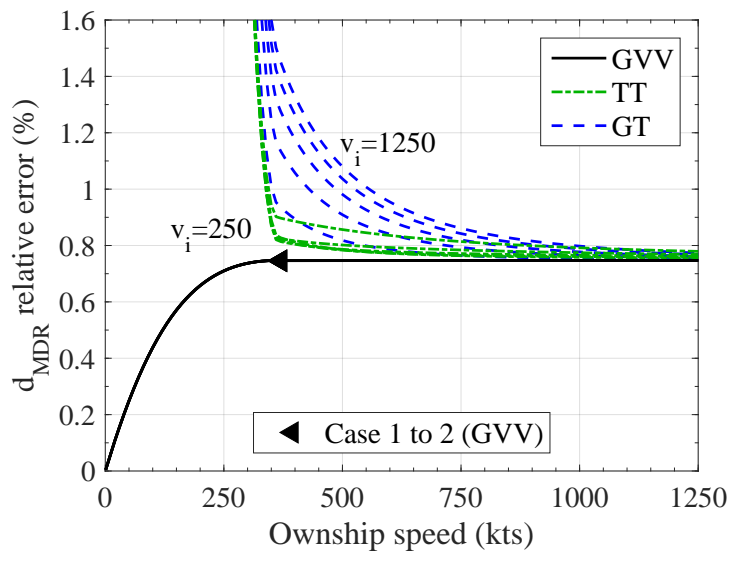

(c) Percent relative error of $d_{\mathrm{MDR}}$ compared to TGVV.

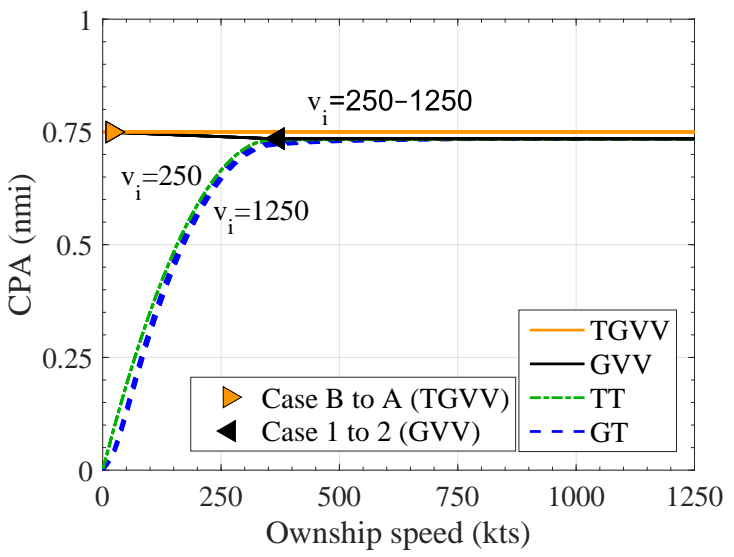

(b) Comparison of the CPA.

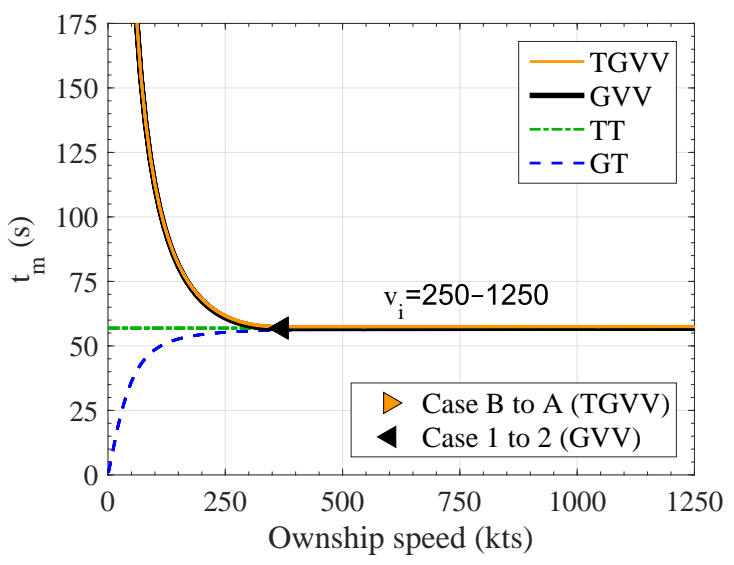

(d) Comparison of $t_{m}$.

Fig. 12 Self-separation results.

parameters. Each of the subplots in Fig. 12 demonstrates that the GVV method can be used as an accurate approximation to the TGVV method when parameter values are aligned with those commonly found in self-separation encounters. Similarly, the TT and GT methods provide good approximations to the TGVV method, but only for large ownship speeds.

Finally, to complete our comparison of the four methods considered in this paper, we present results that characterize the computational cost of each of the methods. These results are shown in Table 4 and include the average runtime of each method and the number of lines of code needed to implement each method. The average runtime for each method was based on 30,000 samples and was executed in Matlab on a 64 bit, $2.70 \mathrm{GHz}$, four-core, Intel I-7 laptop with 16 GB of RAM. For the TGVV method, numerical methods were used with a time step of $0.001 \mathrm{~s}$. The number of lines of code required by each method were determined for a MATLAB implementation. The number 
of lines of code for the TGVV and GVV methods are only approximations because multiple lines of code could be combined; however, this does provide some insight into how much effort would be required to program each method. Although the runtime of each method is of interest, none of the implementations are expensive in terms of required computation time when compared to the timescales of the corresponding DAA maneuvers.

\begin{tabular}{lcccc}
\hline \hline & TGVV & GVV & TT & GT \\
\hline Average runtime $(\mathrm{s})$ & $1.77 \mathrm{e}-2$ & $2.01 \mathrm{e}-5$ & $1.19 \mathrm{e}-6$ & $2.27 \mathrm{e}-6$ \\
Lines of code & $\approx 200$ & $\approx 50$ & 1 & 1 \\
\hline \hline
\end{tabular}

Table 4 Computational cost of each method from average runtime and lines of code.

\section{Conclusion}

The viability of integrating UASs into the National Airspace System is highly dependent on their ability to detect and avoid other air traffic, particularly when flying beyond the line of sight of the operator. For a direct head-on encounter requiring a large detection range, analytical expressions are derived to calculate the minimum detection range required to avoid a predefined safety volume using both dynamic and geometric models of the encounter. The main contribution of this paper is the presentation of two methods used to calculate the minimum detection range: the TGVV and GVV methods. Using the GVV approach, a closed-form analytical expression for the minimum detection range is found by making the assumption that the ownship performs an instantaneous bank-angle maneuver. If this simplifying assumption is removed, the TGVV method can be used to numerically solve for the minimum detection range by using a model of the turning dynamics of the ownship. The minimum detection range calculation takes into account the computation time involved in target tracking, risk assessment, path planning, and pilot response time delay, in addition to the time required to execute the avoidance maneuver.

The TGVV and GVV approaches were compared to the existing TT and GT methods and were shown to create more accurate estimates of the minimum detection range over a wide range of encounter scenarios. For every variation of the encounter parameters, the TGVV method determined a CPA estimate equal to the desired safety radius value, indicating that the true minimum 
detection range had been found. It was also shown that, as the bank-angle dynamics of the ownship became more aggressive, the geometry-based GVV method produced results that approached those of the dynamic-model-based TGVV method. The conditions under which the TT and GT methods produced results that approached the TGVV method were also found to occur at large ownship speeds relative to the intruder speed, fast bank-angle transients, and small bank angles.

Although the direct head-on encounter is important because it requires a large minimum detection range, it would be valuable to extend the minimum detection range methods to a variety of encounter scenarios in addition to the head-on case. Additionally, validation of the minimum detection range results with flight tests to confirm the validity of the underlying assumptions and models would represent a valuable next step in defining integration requirements for UASs.

\section{APPENDIX A: TGVV BANK-ANGLE RESPONSE}

\section{Case A: $\phi_{\max }$ is reached}

Using Fig. 5(a), we see that interval 1 has a positive step in the aileron command; and $t_{0}=0$, $\phi\left(t_{0}\right)=0$, and $\dot{\phi}\left(t_{0}\right)=0$. This results in the following equations for $\phi_{1}(t)$ and $\dot{\phi}_{1}(t)$ :

$$
\begin{aligned}
& \phi_{1}(t)=\tau \dot{\phi}_{\max }\left(e^{-t / \tau}-1\right)+\dot{\phi}_{\max } t \\
& \dot{\phi}_{1}(t)=\dot{\phi}_{\max }\left(1-e^{-t / \tau}\right)
\end{aligned}
$$

Interval 2 has a negative step input with $t_{0}=t_{1}, \phi\left(t_{0}\right)=\phi_{1}\left(t_{1}\right)$, and $\dot{\phi}\left(t_{0}\right)=\dot{\phi}_{1}\left(t_{1}\right)$. This results in the following equations for $\phi 2(t)$ and $\dot{\phi}_{2}(t)$ :

$$
\begin{aligned}
\phi_{2}(t) & =-\tau \dot{\phi}_{\max }\left(e^{-\left(t-t_{1}\right) / \tau}-1\right)-\dot{\phi}_{\max }\left(t-t_{1}\right)+\tau \dot{\phi}_{1}\left(t_{1}\right)\left(1-e^{-\left(t-t_{1}\right) / \tau}\right)+\phi_{1}\left(t_{1}\right) \\
& =\tau \dot{\phi}_{\max }\left(1-2 e^{-\left(t-t_{1}\right) / \tau}+e^{-t / \tau}\right)-\dot{\phi}_{\max }\left(t-2 t_{1}\right) \\
\dot{\phi}_{2}(t) & =-\dot{\phi}_{\max }\left(1-e^{-\left(t-t_{1}\right) / \tau}\right)+\dot{\phi}_{1}\left(t_{1}\right) e^{-\left(t-t_{1}\right) / \tau} \\
& =\dot{\phi}_{\max }\left(2 e^{-\left(t-t_{1}\right) / \tau}-e^{-t / \tau}-1\right)
\end{aligned}
$$

For interval 3, the aileron command is zero. This means the bank angle only experiences the free response due to the initial conditions, and it does not experience any forced response from the 
aileron input. This free response is taken from the terms on the right side of Eqs. (13) and (14) as

$$
\begin{aligned}
& \phi^{f}(t)=\tau \dot{\phi}\left(t_{0}\right)\left(1-e^{-\left(t-t_{0}\right) / \tau}\right)+\phi\left(t_{0}\right), \\
& \dot{\phi}^{f}(t)=\dot{\phi}\left(t_{0}\right) e^{-\left(t-t_{0}\right) / \tau}
\end{aligned}
$$

Using these equations for interval 3 and $t_{0}=t_{2}, \phi\left(t_{0}\right)=\phi_{2}\left(t_{2}\right)$, and $\dot{\phi}\left(t_{0}\right)=\dot{\phi}_{2}\left(t_{2}\right)$ gives the following response for interval 3:

$$
\begin{aligned}
& \phi_{3}(t)=\tau \dot{\phi}_{2}\left(t_{2}\right)\left(1-e^{-\left(t-t_{2}\right) / \tau}\right)+\phi_{2}\left(t_{2}\right), \\
& \dot{\phi}_{3}(t)=\dot{\phi}_{2}\left(t_{2}\right) e^{-\left(t-t_{2}\right) / \tau} .
\end{aligned}
$$

From Fig. 5(a), values for $t_{1}$ and $t_{2}$ are chosen that result in $\phi_{2}\left(t_{2}\right)=\phi_{\max }$ and $\dot{\phi}_{2}\left(t_{2}\right)=0$. With these requirements, the response for interval 3 becomes

$$
\begin{aligned}
& \phi_{3}(t)=\phi_{\max }, \\
& \dot{\phi}_{3}(t)=0 .
\end{aligned}
$$

Interval 4 has a negative step input with $t_{0}=t_{3}, \phi\left(t_{0}\right)=\phi_{3}\left(t_{3}\right)$, and $\dot{\phi}\left(t_{0}\right)=\dot{\phi}_{3}\left(t_{3}\right)$. This results in the following equations for $\phi_{4}(t)$ and $\dot{\phi}_{4}(t)$ :

$$
\begin{aligned}
\phi_{4}(t) & =-\tau \dot{\phi}_{\max }\left(e^{-\left(t-t_{3}\right) / \tau}-1\right)-\dot{\phi}_{\max }\left(t-t_{3}\right)+\tau \dot{\phi}_{3}\left(t_{3}\right)\left(1-e^{-\left(t-t_{3}\right) / \tau}\right)+\phi_{3}\left(t_{3}\right) \\
& =\tau \dot{\phi}_{\max }\left(1-e^{-\left(t-t_{3}\right) / \tau}\right)-\dot{\phi}_{\max }\left(t-t_{3}\right)+\phi_{\max } \\
\dot{\phi}_{4}(t) & =-\dot{\phi}_{\max }\left(1-e^{-\left(t-t_{3}\right) / \tau}\right)+\dot{\phi}_{3}\left(t_{3}\right) e^{-\left(t-t_{3}\right) / \tau} \\
& =\dot{\phi}_{\max }\left(e^{-\left(t-t_{3}\right) / \tau}-1\right)
\end{aligned}
$$

Interval 5 has a positive step input with $t_{0}=t_{4}, \phi\left(t_{0}\right)=\phi_{4}\left(t_{4}\right)$, and $\dot{\phi}\left(t_{0}\right)=\dot{\phi}_{4}\left(t_{4}\right)$. This results in the following equation for $\phi_{5}(t)$ :

$$
\begin{aligned}
\phi_{5}(t) & =\tau \dot{\phi}_{\max }\left(e^{-\left(t-t_{4}\right) / \tau}-1\right)+\dot{\phi}_{\max }\left(t-t_{4}\right)+\tau \dot{\phi}_{4}\left(t_{4}\right)\left(1-e^{-\left(t-t_{4}\right) / \tau}\right)+\phi_{4}\left(t_{4}\right) \\
& =\tau \dot{\phi}_{\max }\left(2 e^{-\left(t-t_{4}\right) / \tau}-1-e^{-\left(t-t_{3}\right) / \tau}\right)+\dot{\phi}_{\max }\left(t-2 t_{4}+t_{3}\right)+\phi_{\max }
\end{aligned}
$$

As stated previously, we must choose values for $t_{1}$ and $t_{2}$ that result in $\phi_{2}\left(t_{2}\right)=\phi_{\max }$ and $\dot{\phi}_{2}\left(t_{2}\right)=0$. To find the values of $t_{1}$ and $t_{2}$, we start with the expression for $\dot{\phi}_{2}(t)$ and the requirement that $\dot{\phi}_{2}\left(t_{2}\right)=0$ to get

$$
0=\dot{\phi}_{\max }\left(2 e^{-\left(t_{2}-t_{1}\right) / \tau}-e^{-t_{2} / \tau}-1\right)
$$


Next, we use the expression for $\phi_{2}(t)$ and the requirement that $\phi_{2}\left(t_{2}\right)=\phi_{\max }$ to get

$$
\phi_{\max }=\tau \dot{\phi}_{\max }\left(1-2 e^{-\left(t_{2}-t_{1}\right) / \tau}+e^{-t_{2} / \tau}\right)-\dot{\phi}_{\max }\left(t_{2}-2 t_{1}\right),
$$

where we notice that the first term on the right side of the equation is the negative of Eq. (A8) multiplied by $\tau$. This cancels the first term, resulting in

$$
\phi_{\max }=-\dot{\phi}_{\max }\left(t_{2}-2 t_{1}\right) .
$$

Rearranging, we get the final expression for $t_{2}$ as

$$
t_{2}=2 t_{1}-\frac{\phi_{\max }}{\dot{\phi}_{\max }}
$$

To find $t_{1}$, we substitute this value of $t_{2}$ back into Eq. A8 and, after algebraic manipulation, we get

$$
0=\left(e^{-t_{1} / \tau}\right)^{2}-2 e^{-t_{1} / \tau}+e^{-\left(\phi_{\max } / \dot{\phi}_{\max }\right) / \tau}
$$

which is a quadratic function in $e^{-t_{1} / \tau}$. Using the quadratic formula, we can find the roots to this equation as

$$
e^{-t_{1} / \tau}=1 \pm \sqrt{1-e^{-\left(\phi_{\max } / \dot{\phi}_{\max }\right) / \tau}}
$$

Rearranging this equation, we can solve for $t_{1}$ as

$$
t_{1}=-\tau \ln \left[1 \pm \sqrt{1-e^{-\left(\phi_{\max } / \dot{\phi}_{\max }\right) / \tau}}\right]
$$

For this expression to give a positive value for $t_{1}$, we must use the negative sign inside the natural logarithm as

$$
t_{1}=-\tau \ln \left[1-\sqrt{1-e^{-\left(\phi_{\max } / \dot{\phi}_{\max }\right) / \tau}}\right] .
$$

Although this equation is mathematically correct, if the quantity $\left(\phi_{\max } / \dot{\phi}_{\max }\right) \tau$ gets too large, numerical roundoff errors can occur while computing the value of $t_{1}$. To help reduce the chance of numerical roundoff error, we multiply the term inside the natural logarithm by its conjugate on the numerator and denominator, which produces the final expression for $t_{1}$ as

$$
t_{1}=-\tau \ln \left[\frac{e^{-\left(\phi_{\max } / \dot{\phi}_{\max }\right) / \tau}}{1+\sqrt{1-e^{-\left(\phi_{\max } / \dot{\phi}_{\max }\right) / \tau}}}\right] .
$$


Now, we finish defining the rest of the time variables $t_{3}, t_{4}$, and $t_{5}$. The time variable $t_{3}$ is defined as

$$
t_{3}=t_{2}+\delta T_{3}
$$

where $\delta T_{3}$ is the total time during interval 3 where the bank angle is at a constant value of $\phi_{\max }$ and can be calculated as

$$
\delta T_{3}=L_{3} / v_{o}
$$

where $L_{3}$ is the length of the path traveled by the ownship during interval 3 and can be calculated as

$$
L_{3}=R_{\min } \delta \chi_{3},
$$

where $\delta \chi_{3}$ is the change in course experienced by the ownship during interval 3 , which can be calculated as

$$
\delta \chi_{3}=\int_{t_{2}}^{t_{3}} \frac{g}{v_{o}} \tan \left(\phi_{3}(t)\right) d t=\chi_{t}-\left(\delta \chi_{1}+\delta \chi_{2}+\left.\delta \chi_{4}\right|_{t_{3}=0, t_{4}=t_{1}}+\left.\delta \chi_{5}\right|_{t_{3}=0, t_{4}=t_{1}, t_{5}=t_{2}}\right),
$$

where $\delta \chi_{1}, \delta \chi_{2}, \delta \chi_{4}$, and $\delta \chi_{5}$ are the changes in course experienced by the ownship during intervals $1,2,4$, and 5 ; and $\chi_{t}$ is the desired change in course during the complete turning maneuver. These variables can be found using Eq. (11) from a coordinated turn as

$$
\begin{aligned}
\delta \chi_{1} & =\int_{0}^{t_{1}} \frac{g}{v_{o}} \tan \left(\phi_{1}(t)\right) d t, \\
\delta \chi_{2} & =\int_{t_{1}}^{t_{2}} \frac{g}{v_{o}} \tan \left(\phi_{2}(t)\right) d t, \\
\delta \chi_{4} & =\int_{t_{3}}^{t_{4}} \frac{g}{v_{o}} \tan \left(\phi_{4}(t)\right) d t, \\
\delta \chi_{5} & =\int_{t_{4}}^{t_{5}} \frac{g}{v_{o}} \tan \left(\phi_{5}(t)\right) d t .
\end{aligned}
$$

The final expression for $t_{3}$ is now found to be

$$
t_{3}=t_{2}+\frac{R_{\min } \delta \chi_{3}}{v_{o}}
$$

where $\delta \chi_{1}, \delta \chi_{2}, \delta \chi_{4}$, and $\delta \chi_{5}$ are defined in Eqs. (A15), (A16), (A17), and (A18). Now that we 
have found $t_{3}$, we can define $t_{4}$ and $t_{5}$ as

$$
\begin{aligned}
& t_{4}=t_{3}+t_{1}, \\
& t_{5}=t_{3}+t_{2} .
\end{aligned}
$$

\section{Case B: $\phi_{\max }$ is not reached}

Similar to case A, we use Eqs. (13) and (14) to define the response of the bank angle to step inputs from the ailerons. Using Fig. 5(b), we see that interval 1 has a positive step, and therefore has the same response to case $\mathrm{A}$ as

$$
\begin{aligned}
& \phi_{1}(t)=\tau \dot{\phi}_{\max }\left(e^{-t / \tau}-1\right)+\dot{\phi}_{\max } t \\
& \dot{\phi}_{1}(t)=\dot{\phi}_{\max }\left(1-e^{-t / \tau}\right)
\end{aligned}
$$

The bank-angle response for intervals 2 and 4 can be combined into a single expression because the aileron input is $-\delta_{a}$ for both intervals. The response for these intervals has the same response as interval 2 in case $\mathrm{A}$ :

$$
\begin{aligned}
& \phi_{2,4}(t)=\tau \dot{\phi}_{\max }\left(1-2 e^{-\left(t-t_{1}\right) / \tau}+e^{-t / \tau}\right)-\dot{\phi}_{\max }\left(t-2 t_{1}\right), \\
& \dot{\phi}_{2,4}(t)=\dot{\phi}_{\max }\left(2 e^{-\left(t-t_{1}\right) / \tau}-e^{-t / \tau}-1\right) .
\end{aligned}
$$

Interval 5 differs from case A because the previous interval 4 has been combined with interval 2. For interval 5 of this case, we also have a positive step and $t_{0}=t_{4}$; however, $\phi\left(t_{0}\right)=\phi_{2,4}\left(t_{4}\right)$, and $\dot{\phi}\left(t_{0}\right)=\dot{\phi}_{2,4}\left(t_{4}\right)$. This results in the following equation for $\phi_{5}(t)$ :

$$
\begin{aligned}
\phi_{5}(t) & =\tau \dot{\phi}_{\max }\left(e^{-\left(t-t_{4}\right) / \tau}-1\right)+\dot{\phi}_{\max }\left(t-t_{4}\right)+\tau \dot{\phi}_{2,4}\left(t_{4}\right)\left(1-e^{-\left(t-t_{4}\right) / \tau}\right)+\phi_{2,4}\left(t_{4}\right), \\
& =\tau \dot{\phi}_{\max }\left(2 e^{-\left(t-t_{4}\right) / \tau}-2 e^{-\left(t-t_{1}\right) / \tau}+e^{-t / \tau}-1\right)+\dot{\phi}_{\max }\left(t+2 t_{1}-2 t_{4}\right) .
\end{aligned}
$$

In deriving $t_{1}$ for case $\mathrm{A}$, we used the constraint $\phi_{2}\left(t_{2}\right)=\phi_{\max }$; however, this constraint is not true for case B. Finding $t_{1}$ for this case requires a more in-depth analysis. From the total turning maneuver of the ownship for case $\mathrm{B}$, we know that

$$
\chi_{t}=\delta \chi_{1}+\delta \chi_{2,4}+\delta \chi_{5},
$$


where $\delta \chi_{1}$ and $\delta \chi_{5}$ were defined in Eqs. (A15) and (A18) in case A, and $\delta \chi_{2,4}$ is the change in course experienced by the ownship during the combined intervals 2 and 4 as

$$
\delta \chi_{2,4}=\int_{t_{1}}^{t_{4}} \frac{g}{v_{o}} \tan \left(\phi_{2,4}(t)\right) d t .
$$

Moving $\chi_{t}$ to the right side of the equation results in

$$
0=\delta \chi_{1}+\delta \chi_{2,4}+\delta \chi_{5}-\chi_{t}
$$

To find $t_{1}$, we express each of the components of Eq. (A26) in terms of the variable $t_{1}$ and use the Newton-Raphson method to find the value of $t_{1}$ that makes the equation equal zero. The variables that need to be expressed in terms of $t_{1}$ are $t_{2}, t_{4}$, and $t_{5}$.

A constraint from case A that we can use for case B is $\dot{\phi}_{2}\left(t_{2}\right)=0$. This constraint resulted in Eq. (A8), which can be solved for $t_{2}$ in terms of $t_{1}$ as

$$
t_{2}=\tau \ln \left[2 e^{t_{1} / \tau}-1\right]
$$

Using Fig. 5(b) and Eq. (A27), we create expressions for $t_{4}$ and $t_{5}$ in terms of $t_{1}$ as

$$
\begin{aligned}
& t_{4}=t_{2}+t_{1}=t_{1}+\tau \ln \left[2 e^{t_{1} / \tau}-1\right], \\
& t_{5}=2 t_{2}=2 \tau \ln \left[2 e^{t_{1} / \tau}-1\right] .
\end{aligned}
$$

Using the expression for $t_{4}$, we modify Eq. (A24) to be expressed in terms of $t_{1}$ as

$$
\phi_{5}(t)=\tau \dot{\phi}_{\max }\left(e^{-t / \tau}\left(2 e^{t_{1} / \tau}-1\right)^{2}-1-2 \ln \left[2 e^{t_{1} / \tau}-1\right]\right)+\dot{\phi}_{\max } t
$$

\section{ACKNOWLEDGMENTS}

This research was conducted in the Center for Unmanned Aircraft Systems (C-UAS) with support from the National Science Foundation Industry/University Cooperative Research Center program grant number IIP-1161036 and C-UAS Industry Advisory Board members. 


\section{REFERENCES}

[1] RTCA-SC-228, "Draft Detect and Avoid (DAA) Minimum Operational Performance Standards for Verification and Validation." Tech. rep., Radio Telecommunications Corporation of America, Washington D.C., 2015.

[2] Contarino, V. M. and Scire Consultants, L., "All Weather Sense and Avoid System for UASs," Tech. rep., Report to the Office of Naval Research, 2009.

[3] Dalamagkidis, K., Valavanis, K. P., and Piegl, L. A., On Integrating Unmanned Aircraft Systems into the National Airspace System, Vol. 52, Springer Science+Business Media B.V., 2nd ed., 2012.

[4] Angelov, P., Sense and Avoid in UAS: Research and Applications, John Wiley \& Sons, Ltd., 2012.

[5] Prats, X., Luisn, D., Jorge, R., Pablo, R., and Enric, P., "Requirements, Issues, and Challenges for Sense and Avoid in Unmanned Aircraft Systems," Aircraft, Vol. 49, No. 3, 2012, pp. 677-687.

[6] Geyer, C., Singh, S., and Chamberlain, L., "Avoiding Collisions Between Aircraft: State of the Art and Requirements for UAVs operating in Civilian Airspace," Tech. Rep. CMU-RI-TR-08-03, Robotics Institute, Carnegie Mellon University, 2008.

[7] Boskovic, J. D., Jackson, J. A., and Mehra, R. K., "Sensor and Tracker Requirements Development for Sense and Avoid Systems for Unmanned Aerial Vehicles," in "AIAA Modeling and Simulation Technologies (MST) Conference," Boston, MA, 2013.

[8] Melnyk, R., Daniel, S., Vitali, V., and Hernando, J., "Sense and Avoid Requirements for Unmanned Aircraft Systems Using a Target Level of Safety Approach," Risk Analysis, Vol. 34, No. 10, 2014, pp. $1894-1906$.

[9] Sahawneh, L. R., Spencer, J., Beard, R. W., and Warnick, K. F., "Minimum Required Sensing Range for UAS Sense and Avoid Systems," in "AIAA Infotech@Aerospace," AIAA, San Diego, California, 2016.

[10] Lee, S. M., Park, C., Johnson, M. A., and Mueller, E. R., "Investigating Effects of Well Clear Definitions on UAS Sense-And-Avoid Operations," in "Aviation Technology, Integration, and Operations Conference," AIAA, Los Angeles, CA, 2013.

[11] Consiglio, M., Chamberlain, J., Munoz, C., and Hoffler, K., "Concept Of Integration For UAS Operations In The NAS," in "28th International Congress of the Aeronautical Sciences (ICAS)," Brisbane, Australia, 2012.

[12] FAA SAA Workshop, "FAA Workshop on Sense and Avoid (SAA) for Unmanned Aircraft Systems (UAS) Report," October, 2009.

[13] Cook, S. P., Brooks, D., Cole, R., Hackenberg, D., and Raska, V., "Defining Well Clear for Unmanned 
Aircraft Systems," AIAA Infotech @ Aerospace, Vol. January, 2015, pp. 1-20, doi:10.2514/6.2015-0481.

[14] Beard, R. W. and McLain, T. W., Small Unmanned Aircraft: Theory and Practice, Princeton University Press, 2012.

[15] Erzberger, H. and Heere, K., "Algorithm and operational concept for resolving short-range conflicts," Proceedings of the Institution of Mechanical Engineers Part G-Journal of Aerospace Engineering, Vol. 224, No. G2, 2010, pp. 225-243, doi:Doi10.1243/09544100jaero546.

[16] Weisstein, E. W., "Cubic Formula," From MathWorld-A Wolfram Web Resource. URL: http:// mathworld.wolfram. com/CubicFormula.html. [cited: 2017-01-18]. 